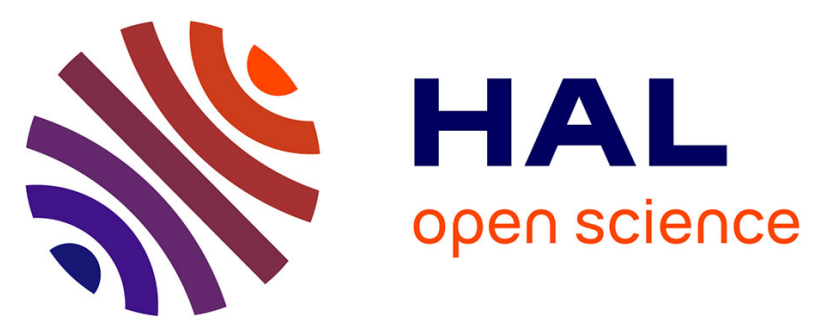

\title{
Review of the Current Literature and Our Experience on the Value of OCT-angiography in White Dot Syndromes
}

Céline Mebsout-Pallado, Raphaëlle Orès, Céline Terrada, Kunal Dansingani, Jay Chhablani, Andrew Eller, Joseph N Martel, Alexander Anetakis, Jean Harwick, Evan Waxman, et al.

\section{To cite this version:}

Céline Mebsout-Pallado, Raphaëlle Orès, Céline Terrada, Kunal Dansingani, Jay Chhablani, et al.. Review of the Current Literature and Our Experience on the Value of OCT-angiography in White Dot Syndromes. Ocular Immunology and Inflammation, 2021, pp.1-15. 10.1080/09273948.2020.1837185. hal-03182427

\section{HAL Id: hal-03182427 https: / hal.sorbonne-universite.fr/hal-03182427}

Submitted on 26 Mar 2021

HAL is a multi-disciplinary open access archive for the deposit and dissemination of scientific research documents, whether they are published or not. The documents may come from teaching and research institutions in France or abroad, or from public or private research centers.
L'archive ouverte pluridisciplinaire HAL, est destinée au dépôt et à la diffusion de documents scientifiques de niveau recherche, publiés ou non, émanant des établissements d'enseignement et de recherche français ou étrangers, des laboratoires publics ou privés. 


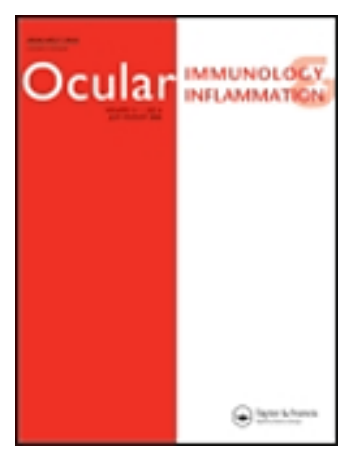

\section{Review of the current literature and our experience on the value of OCT-angiography in white dot syndromes}

\begin{tabular}{|c|c|}
\hline Journal: & Ocular Immunology and Inflammation \\
\hline Manuscript ID & NOII-2020-0038.R2 \\
\hline Manuscript Type: & Invited Review \\
\hline $\begin{array}{r}\text { Date Submitted by the } \\
\text { Author: }\end{array}$ & 27-Sep-2020 \\
\hline Complete List of Authors: & $\begin{array}{l}\text { Mebsout-Pallado, Céline; Quinze-Vingts National Eye Hospital, UPMC- } \\
\text { Sorbonne Universities, Paris, France. } \\
\text { Ores, Raphaëlle; Quinze-Vingts National Eye Hospital, UPMC-Sorbonne } \\
\text { Universities, Paris, France. } \\
\text { Terrada, Céline; Pitié-Salpêtrière Hopital, Ophthalmology } \\
\text { Dansingani, Kunal; Department of Ophthalmology, University of } \\
\text { Pittsburgh School of Medicine, } \\
\text { Chhablani, Jay K; Department of Ophthalmology, University of Pittsburgh } \\
\text { School of Medicine, } \\
\text { Eller, Andrew; University of Pittsburgh Medical Center, Ophthalmology } \\
\text { Martel, Joseph; Department of Ophthalmology, University of Pittsburgh } \\
\text { School of Medicine, } \\
\text { anetakis, Alexander; Department of Ophthalmology, University of } \\
\text { Pittsburgh School of Medicine, } \\
\text { Harwick, Jean; Department of Ophthalmology, University of Pittsburgh } \\
\text { School of Medicine, } \\
\text { Waxman, Evan; University of Pittsburgh Medical Center, Ophthalmology } \\
\text { Gallagher, Denise; Department of Ophthalmology, University of } \\
\text { Pittsburgh School of Medicine, } \\
\text { Prensky, Colin; Department of Ophthalmology, University of Pittsburgh } \\
\text { School of Medicine, } \\
\text { Indermill, Chad; Department of Ophthalmology, University of Pittsburgh } \\
\text { School of Medicine, } \\
\text { Sedira, Neila; Quinze-Vingts Hospital, Department of Internal Medicine } \\
\text { Héron, Emmanuel; Quinze-Vingts National Eye Hospital, UPMC-Sorbonne } \\
\text { Universities, Paris, France. } \\
\text { Paques, Michel; Quinze-Vingts National Ophthalmology Hospital, } \\
\text { Ophthalmology IV } \\
\text { Brignole-Baudouin, Françoise ; Quinze-Vingts Hospital, DHU Sight } \\
\text { Restore, Laboratory; Universite Paris Descartes } \\
\text { Bodaghi, Bahram; Hopital Pitie-Salpetriere } \\
\text { Sahel, José-Alain; Centre Hospitalier National d'Ophtalmologie des }\end{array}$ \\
\hline
\end{tabular}


Quinze-Vingts. Ophthalmology Department and Laboratory and DHU Sight Restore; Sorbonne Universités, UPMC Univ Paris VI Gaudric, Alain; Department of Ophthalmology, Lariboisière Hospital, Université Paris Diderot, Sorbonne

Mrejen, Sarah; Quinze-Vingts National Eye Hospital, UPMC-Sorbonne Universities, Paris, France.

Errera, Marie-Helene; Quinze-Vingts National Eye Hospital, UPMCSorbonne Universities, Paris, France.,

Birdshot chorioretinopathy, multifocal choroiditis, multifocal placoid Keywords: pigment epitheliopathy, MEWDS, OCT-A, serpiginous-like choroiditis, serpiginous choroiditis, syphilitic placoid chorioretinitis, uveitis

\section{SCHOLARONE ${ }^{m}$ Manuscripts}




\section{Review of the current literature and our experience on the value of OCT- angiography in white dot syndromes}

Céline Mebsout-Pallado, ${ }^{1}$ Raphaëlle Orès, ${ }^{1}$ Céline Terrada, ${ }^{2,3}$ Kunal K. Dansingani, ${ }^{4}$ Jay

Chhablani, ${ }^{4}$ Andrew W. Eller, ${ }^{4}$ Joseph N. Martel, ${ }^{4}$ Alexander Anetakis, ${ }^{4}$ Jean C. Harwick, ${ }^{4}$

Evan L. Waxman, ${ }^{4}$ Denise S. Gallagher, ${ }^{4}$ Colin Prensky, ${ }^{4}$ Chad Indermill, ${ }^{4}$ Neila Sedira, ${ }^{1}$

Emmanuel Héron, ${ }^{1}$ Michel Paques, ${ }^{1,5}$ Françoise Brignole-Baudouin, ${ }^{1,6}$ Bahram Bodaghi, ${ }^{7}$ José-

Alain Sahel, ${ }^{1,4,5}$ Alain Gaudric, ${ }^{2}$ Sarah Mrejen, ${ }^{1}$ Marie-Hélène Errera, ${ }^{1,4,5}$

10

1. Centre Hospitalier National des Quinze-Vingts, DHU Sight Restore, Paris, France

2. Department of Ophthalmology, Lariboisière Hospital, Université Paris Diderot, Sorbonne, Paris, France

3. Centre Ophtalmologique Roule Peretti, 169 avenue Achille Peretti, 92200 Neuilly sur Seine, France

4. Department of Ophthalmology, University of Pittsburgh School of Medicine, Pittsburgh, Pennsylvania, USA

5. INSERM-DHOS CIC, Sorbonne Universités, UPMC Univ Paris VI, France

6. Université Paris Descartes (Sorbonne Paris Cité), Paris V, France

7. Department of Ophthalmology, Hopital Pitié-Salpêtrière, DHU Sight Restore, Sorbonne Universités, UPMC Univ Paris VI, France

Corresponding author: Marie-Hélène Errera, UPMC Eye Center, University of Pittsburgh School of Medicine, Pittsburgh, Pennsylvania. USA

$$
\text { erreram@upmc.edu }
$$

Abstract: 150 words

Words: 5240 


\section{Abstract}

3 Purpose: The aim of this study is to describe the application of OCT-A in various posterior

$4 \quad$ uveitis disorders in our experience and to compare it with the available literature.

5 Methods: Literature searches were performed using electronic medical databases on

6 posterior uveitis and OCT-A. Eighteen eyes (16 patients) with the following diagnoses:

7 multifocal choroiditis (MFC), multifocal placoid pigment epitheliopathy (APMPPE), multiple

8 evanescent white dot syndrome (MEWDS), tuberculous serpiginous-like choroiditis (SLC),

9 serpiginous choroiditis (SC), and birdshot chorioretinopathy (BSCR) were studied.

10 Results: We found flow void of the choriocapillaris in patients with APMPPE, SC, MFC, BSCR

11 and in SLC. In contrast, perfusion of the choriocapillaris seemed normal in patients with 12 MEWDS.

13 Conclusions: We confirmed that OCT-A contributes new information on the physiopathology 14 of white dot syndromes and other inflammatory chorioretinopathies, notably on whether or 15 not the choriocapillaris is involved. Comparing the OCTA features of those entities allowed 16 us to suggest that both entities APMPPE and SLC might be part of the same spectrum of 17 inflammatory disease with primary involvement of the level of the choriocapillaris and 18 secondary RPE damage.

Keywords: Birdshot chorioretinopathy, multifocal choroiditis, multifocal placoid pigment epitheliopathy, MEWDS, OCT-A, serpiginous-like choroiditis, serpiginous choroiditis, uveitis, No potential conflict of interest was reported by the authors. 


\section{INTRODUCTION}

2 The term "white dot syndromes" is used to refer to a heterogenous group of chorioretinal

3 inflammatory diseases. Apart from grouping these conditions and reminding us that they can

4 feature multiple inflammatory lesions in various configurations, the term "white dot

5 syndrome" is of limited value as it does not convey useful information about etiology,

6 classification or treatment of these conditions. In addition, other uveitis may take on the appearance of white spot syndrome such as those associated with tuberculosis or syphilis for example. It is important to try to distinguish the etiologies underlying "white dot syndromes" because their causes may vary and their treatments differ. Some patients can develop a clinical picture which resembles more than one type of inflammatory process, suggesting that overlap can occur between different inflammatory disease entities. One example of this is multifocal choroiditis (MFC) and multiple evanescent white dot syndrome (MEWDS) occurring simultaneously. Another example is MFC that is complicated by acute zonal occult outer retinopathy (AZOOR)..$^{1-3}$

From the pathophysiological point of view, patterns of tissue involvement also seem to differ among "white dot syndromes." Certain entities seem primarily to involve the retinal pigmentary epithelium (RPE) while others seem to show predilection for the choriocapillaris. Furthermore, the development of new imaging modalities sometimes reveals characteristics that require etiological hypotheses to be revised. For example, Gass originally described multifocal placoid pigment epitheliopathy (APMPPE) in terms of RPE involvement, but more recent multimodal imaging studies seem to place its primitive origins in the choriocapillaris. ${ }^{4-}$ 6

New imaging techniques make it possible to describe different signs corresponding to different pathophysiological mechanisms. Indocyanine green angiography (ICGA) can analyze choroidal vascularization more precisely than can fluorescein angiography (FA). However, ICGA cannot provide a topographic layer-by-layer analysis of the retina and the choroid, and 
1 The recent use of en face OCT, which provides depth-resolved layer-by-layer analysis of the

2 retina and the choroid, and OCT-A which can image vessels based on motion contrast, have

3 added new informations regarding the pathophysiological processes involved in MEWDS and

4 in other white dot syndromes. ${ }^{7}$ OCTA integrated into a multimodal approach has already

5 helped distinguish between inflammatory and neovascular lesions in MFC. ${ }^{4,8-11}$ Also, OCT-A

6 seems to be a tool that can refine the analysis of the choriocapillaris.

7 This objective of this review was to review the available literature on the physiopathology of

8 white dot syndromes and posterior uveitis through the contribution of OCT-A and to

9 incorporate our experience into the current knowledge. The retinochoroidal

10 microvasculature was therefore studied using OCT-A in a selection of cases with

11 inflammatory white dot syndrome or inflammatory chorioretinopathies at initial

12 presentation.

\section{MATERIAL AND METHODS}

Literature searches were performed using PubMed as the database for the electronic literature search on the OCT-A features of the following entities: white dot syndromes, APMPPE, MEWDS, tuberculosis (TB) -related serpiginous-like choroiditis (SLC), serpiginous choroiditis (SC), punctate inner choroiditis (PIC), MFC, birdshot chorioretinopathy (BSCR), and posterior uveitis. In addition, the bibliographies of existing literature reviews and key articles, were reviewed to identify other relevant articles appropriate for inclusion. The key search question being : "Evaluation of the pathophysiology of uveitis by OCTA." The goal of the literature search strategy was to identify published articles for which the topic of interest was the primary focus, rather than all articles on the topic. Internet searches provided supplemental information, thus ensuring that interpretation of the identified articles was consistent with current knowledge. We excluded studies that were not published in the English language and those that did not report research results related to the key question.

We described the OCT-A findings in a selection of cases presenting consecutively for uveitis consultation at the Quinze-Vingts National Ophthalmology Hospital, Paris, France, between May 2016 and March 2017, with white dot syndrome and/or inflammatory chorioretinopathy in the acute phase. The data set was subsequently expanded to include 
1 patients seen at in UPMC Eye Center, Pittsburgh, PA, USA and in Hopital Pitié-Salpêtrière,

2 Paris between February 2019 and November 2019. All imaging results were reviewed by the

3 authors. Disagreements in interpretation were reconciled by one of the authors (S.M.).

4 Ophthalmic findings at the time of presentation were recorded, including best-corrected

5 visual acuity and findings on slit-lamp examination and dilated fundus examination.

6 Fundus Photography Color and monochromatic red-free photographs were acquired using a

7 Topcon TRC-50IX fundus camera (Topcon Medical Systems, Tokyo, Japan). Near-infrared

8 reflectance images were acquired with the Heidelberg Spectralis system (HRA Heidelberg

9 Engineering, Heidelberg Germany) using a $30^{\circ}$ square field of view at a resolution of 1,536

10 square pixels following the standard procedure for image acquisition including focus of the

11 retinal image in the infrared reflection mode with an 820-nm wavelength. In the same

12 sitting, a $30^{\circ} \times 30^{\circ}$ BluePeak laser fundus autofluorescence (FAF) image was captured.

13 Acute, early, FA and ICGA of the posterior pole and the nine peripheral fields were obtained

14 using a confocal scanning laser ophthalmoscope (Spectralis-HRA +OCT; Heidelberg

15 Engineering) for all patients. FA was obtained after intravenous injection of $10 \mathrm{~mL}$ of $5 \%$

16 fluorescein dye and ICGA images were acquired after intravenous injection of $50 \mathrm{mg}$ of 17 indocyanine green dye.

18 Spectral domain optical coherence tomography (SD-OCT) imaging was performed with the 19 Spectralis HRA + OCT (Heidelberg Engineering). This equipment provided simultaneous OCT 20 scans and near-infrared reflectance, short-wavelength fundus autofluorescence (SW-AF), FA, 21 or ICGA imaging. Subsequent image superimposition allowed point-to-point correlation 22 between the en-face and cross-sectional images. The OCT imaging was acquired with a 23 broadband 870-nm superluminescent diode that scanned the retina at 40,000 A-scans per 24 second with an optical axial depth resolution of $7 \mu \mathrm{m}$. The standard protocol included at 25 least 12 OCT scans averaged to reduce the signal-to-noise ratio by a factor of 5 and at least 26 one 9-mm horizontal line scan through the fovea (volume acquisition, $6 \mathrm{~mm} \times 6 \mathrm{~mm}$; 60 27 scans (dense)).

28 Enhanced-depth imaging OCT was acquired following the methodology previously described 29 in the literature. ${ }^{12}$

30 En-face macular mapping was obtained with Optovue OCT, three-dimensional $6 \times 6-\mathrm{mm}$ 31 macular cube raster scans with $400 \times 400$ axial scans were obtained. For each patient, two 32 volumetric scans with orthogonal fast scan directions were acquired, processed with 
1 software for motion correction, and merged into a single volumetric data set to increase the

2 signal. Scans were repeated during the early and late recovery phase. The Optovue RTVue

3 XR Avanti (Optuvue, Inc.) was used to obtain OCTA images. A 3×3-mm scanning area

4 centered on the fovea was captured for blood flow measurements. In the fast transverse

5 scanning direction, 200 axial scans were sampled along a 3-mm region to obtain a single B-

6 scan. Eight consecutive B-scans (M-B frames) were captured at a fixed position before

7 proceeding to the next sampling location. A total of 200 locations along a 3-mm region in the 8 slow transverse direction were sampled to form a 3D data cube. With a B-scan frame rate of

9455 frames per second, the 1,600 B-scans in each scan were acquired in approximately $3.5 \mathrm{~s}$.

10 Four volumetric raster scans, including two horizontal priority fast transverse ( $x$-fast) scans

11 and two vertical priority fast transverse ( $y$-fast) scans, were obtained consecutively in one

12 session. The SSADA algorithm was used to distinguish blood flow from static tissue.

13 We also performed a $3 \times 3,6 \times 6 \mathrm{~mm}$ and $12 \times 12 \mathrm{~mm}$ acquisition on the retina with the OCT-A 14 Angioplex (Zeiss) for 3 patients. This OCT-A uses an algorithm known as OCT

15 microangiography-complex, which incorporates differences in both the phase and intensity

16 information contained within sequential B-scans at the same position. ${ }^{13}$

17 OCT-A was performed in all patients' eyes at initial presentation, and at final presentation in 18 one patient's eye with APMPPE, in 4 patients' eyes with MFC, and in 2 patients with BSCR 19 with a follow-up duration from one month up to eight months.

20 The OCT-A findings in the selection of our cases was compared to the available literature and 21 applied to the understanding of the physiopathology of white dot syndromes and posterior 22 uveitis.

This study was conducted in accordance with the Declaration of Helsinki and was 25 approved by the Ethics Committee of the French Society of Ophthalmology and by the 26 University of Pittsburgh IRB\#: PRO18020162.

RESULTS

29 Clinical features and Imaging 
1 This study included 18 eyes (16 subjects; 8 females) diagnosed with "white dot syndromes"

2 evaluated at presentation before treatment: APMPPE ( $n=2$ patients, 2 eyes), MEWDS ( $n=3$

3 patients, 3 eyes), tuberculosis (TB) -related serpiginous-like choroiditis (SLC) ( $n=1$ patients, 1

4 eye), serpiginous choroiditis ( $n=1$ patient, 1 eye), MFC ( $n=7$ patients, 7 eyes), BSCR ( $n=2$

5 patients, 4 eyes). The mean age of the subjects was $38 \pm 18$ years (range, 12- 72 years).

6 Table 1 shows demographics and the retinal imaging testings performed, as well as the any 7 treatments initiated.

8 Patients were evaluated through follow-up after treatment. Table 2 shows the OCT-A

9 findings before and during follow-up, when performed.

10

\section{- Two patients with APMPPE}

The fundus colour photograph showed deep white lesions distributed in the posterior pole and mid-periphery in two cases. The APMPPE lesions were mainly located in the posterior pole but spreading outside the vascular arcades. FAF showed hyperautofluorescent lesions co-localizing with the white lesions seen in the fundus colour photograph (Figure 1). FA showed an early hypofluorescent lesion and then hyperfluorescence at the intermediate and late frames. ICGA showed multiple hypofluorescent lesions at the early and late frames. SD-OCT showed IS-OS layer disruption and thickened areas as well as outer retinal hyperreflectivity.

OCT-A showed no flow abnormalities at the superficial and deep retinal plexuses of the patients with APMPPE; at the level of the choriocapillaris, they presented areas of hypoperfusion on OCT-A, which were superimposed on the areas which were hypofluorescent in the early frames of FA and ICGA, and then hyperfluorescent in the late frames of FA. Comparing the lesions on OCT-A, the en face OCT and the hypoautofluorescent areas, we observed that the circulatory defects of the choriocapillaris seen on OCT-A and the en face OCT were more extensive than the lesions of the RPE seen on FAF, which demonstrates that they are not optical masking artifacts or shadows, but rather occlusive vasculitis lesions with the primitive origin in the choriocapillaris (Figure 2', supplemental material).

En-face OCT in two patients with APMPPE showed hyporeflective areas at the outer retina (asterisks Figure 2) and hyper-reflective dots at the choriocapillaris. 
1 During the follow-up 2 months after systemic corticosteroid therapy, in the plane of the

2 choriocapillary layer, on OCT-A we observed regression of the hypoperfusion areas of the

3 choriocapillaris (Figure 2', supplemental material).

4 In multimodal imaging, two different classes of APMPPE lesions appeared, which also 5 seemed to progress differently.

6 The first were lesions that were visible at the early and late frames of the FA as well as on

7 autofluorescence. These same lesions appeared as hypoperfusion of the choriocapillaris on

8 OCT-A (shown as a red circle in Figure 2'). These lesions seemed to be related to RPE lesions,

9 and they showed up as scars on SD-OCT during progression.

10 In addition, the second type of lesion appeared either isoautofluorescent or

11 hyperautofluorescent on FAF imaging (shown as yellow circles in Figure 2'). These lesions

12 appeared to be hypofluorescent on FA at the early frames and were nonapparent at the late

13 frames of FA, whereas they were hyporeflective on OCT-A. The latter lesions were self-

14 limiting under corticosteroid treatment and seemed to be related to the more extensive

15 occlusive vasculitis lesions of the choriocapillaris, and were potentially reversible (Figure 2').

\section{- Three patients with MEWDS}

18 Fundus examination of the affected eyes demonstrated deep yellow or white spots in the posterior pole in two eyes, spreading to the midperiphery in one eye.

20 On FAF the lesions were hyperautofluorescent and more numerous than on clinical

21 examination. Some subclinical lesions were detected on FAF.

22 On FA, early frames showed wreath-like hyperfluorescence of lesions that persisted into the 23 late frames. ICG angiography showed hypofluorescent spots at these locations in late 24 frames.

25 SD-OCT showed disruption of photoreceptors, most particularly the ellipsoid zone and 26 interdigitation zone, in areas corresponding to individual lesions.

27 En-face OCT showed hyper-reflective dots in the outer nuclear layer (ONL). En-face OCT 28 centered on the ellipsoid zone showed hyporeflective areas corresponding to ellipsoid layer 29 disruption and were superimposed on hypofluorescent lesions visible in the late frames of 30 ICGA (Figure 3).

31 OCT-A of the superficial and deep retinal capillary plexus and the choriocapillaris and en-face 32 OCT-A of the choriocapillaris were normal in these 3 patients (Figure 4). 
- $\quad$ APMPPE versus MEWDS

3

4

OCT-A showed hypoperfusion of the choriocapillaris in two patients with APMPPE, contrary to those who were diagnosed with MEWDS. Hypoperfusion of the choriocapillaris on OCT-A has already been demonstrated in the literature in patients with APMPPE. 8,14

The hypofluorescence of APMPPE in the early phase of the FA and throughout the ICGA sequence suggests multifocal choroidal hypoperfusion as the cause of the opacification of the outer retina corresponding to its hyper-reflectivity on SD-OCT.

We observed that hypofluorescent lesions in the late frames of ICGA in MEWDS are superimposed on hyporeflective lesions on en-face OCT centered on the ellipsoid and correspond to ellipsoid disruption areas on SD-OCT.

\section{- One patient with TB-related serpiginous-like choroiditis (SLC)}

Fundus examination showed multiple irregular serpiginous lesions involving the posterior pole and periphery. On FAF, the lesions presented as hyperautofluorescent.

FA showed the hyperfluorescence at intermediate frames more accurately. The hyperfluorescence was increased in the later frames. ICGA showed confluent hypofluorescent lesions in the early and late frames.

SD-OCT demonstrated areas of IS/OS layer disruption and areas of hyperreflectivity of the RPE, atrophy of the outer retina with cystoid edema, and hyperreflectivity of the choroid (Figure 5). En-face OCT revealed hyporeflective areas at the outer retina and hyperreflective dots of the choriocapillaris (patient 6).

The superficial and deep plexuses on OCT-A showed no anomalies. OCT-A showed hypoperfusion areas of the choriocapillaris (or choriocapillaris flow void areas).

Superimposition between the choriocapillaris hypoperfusion lesions on OCT-A and the hyperfluorescent lesions on FA, hypofluorescent lesions on ICGA were noted (Figure 5), as described by other authors. ${ }^{15,16}$ OCT-A showed areas of possible flow void at the level of the choriocapillaris. 
1 SD-OCT found outer retinal hyper-reflective deposits with knob-like elevations of RPE;

2 ellipsoid and ELM disruption, atrophy of RPE-Bruch's complex, increased choroidal

3 reflectance, choroidal thinning, and loss of choriocapillaris.

4 - APMPPE versus tuberculosis (TB) - related SLC

5 In both APMPPE and SLC, we observed more extensive areas of choriocapillaris

6 hypoperfusion on OCT-A than RPE lesions on FAF. We believe that these are two separate

7 entities with different pathogenesis but with similar layers of inflammatory involvement. SLC

8 presents as a more extensive disease at baseline compared to APMPPE with more confluent

9 areas of choriocapillaris ischemia on OCT-A. And SLC has a more severe progressive and

10 sometimes recurrent chronic course compared to APMPPE, with more extensive RPE

11 damage.

12 Moreover, in both entities, at final exam, there were areas where the initial lesions had

13 completely resolved without any RPE atrophy and areas where there was a legacy of

14 complete RPE atrophy. On FAF, the areas of RPE atrophy were more widespread in SLC than 15 in APMPPE at both baseline and final exam.

- One patient with serpiginous choroiditis

18 The fundus examination showed serpiginous lesions of different ages.

19 FA showed hypofluorescent confluent lesions in the early frames, which became 20 hyperfluorescent on the late frames with incomplete hyperfluorescent edges indicating 21 active disease. ICGA showed these same lesions as remaining hypofluorescent throughout 22 the sequence (on the early and late frames (Figure $6^{\prime}$ )). SD-OCT found areas with atrophy of 23 the outer retina and ellipsoid disruption.

24 OCT-A showed no flow abnormalities of the superficial and deep plexuses and the outer 25 retina. There were hypoperfusion lesions of the choriocapillaris ${ }^{8}$, suggesting choriocapillary 26 ischemia in this disease. ${ }^{17}$ The hypoperfusion areas of the choriocapillaris on OCT-A were 27 superimposed with the hyperfluorescent lesions in the late frame of FA (Figure 6) and the 28 hypofluorescent areas on ICGA. 
1 Fundus color showed yellowish focal lesions. FAF showed multiple areas of

2 hypoautofluorescence corresponding to the areas of lesions on ophthalmoscopy.

3 FA showed the focal lesions to be hypofluorescent in the early and hyperfluorescent in the

4 late stages of the angiogram. ICGA showed rounded hypofluorescent lesions.

5 SD-OCT showed of localized RPE elevations with underlying hyporeflective space and sub-

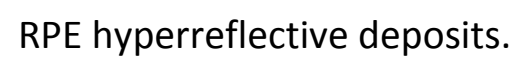
follow-up, after IV solumedrol and oral prednisone treatment. The other patient (patient 11) showed an hypoperfusion area appearing less extensive on OCT-A after a course of oral prednisone (final images not shown).

\section{- Four patients with choroidal neovascularization (CNV) associated with MFC/ PIC}

Fundus color showed a yellow subretinal lesion. FAF showed an area of hypoautofluorescence in the same area. FA evidenced a hyperfluorescent lesion with late staining. SD-OCT revealed a subretinal lesion associated with subretinal fluid in 3 patients. OCT-A showed flow signal in the morphology of a CNV lesion at the outer retina and the choriocapillaris. ICGA demonstrated late hyperfluorescence of the CNV surrounded by a hypofluorescent border that was superimposed on the vascular network visualized on OCT-A (Figure 7). One patient's eye (with MFC and CNV) had repeated OCT-A imaging showing mixed CNV membrane enlargement when having a recurrence. Each relapse was treated with monthly anti-VEGF injections and triamcinolone subtenon injections every 3 months. Another patient's eye had no sign of vascular network one month after a single anti-VEGF intravitreal injection for CNV related to PIC, though (Figure 7').

\section{- Two patients with BSCR}

We imaged the eyes of one patient with recent symptoms related to uveitis and before any treatment for BSCR. Reflectivity of both the EZ and the IZ at SD-OCT B-scan passing through the foveas were altered or reduced (Figure 8').

The other patient who had imaging for BSCR had been known with the disease for the last 12 years and BSCR was inactive currently under systemic immunosuppressive treatment. FA 
1 showed hyperfluorescent foci from early frame to late frames. With ICGA, these lesions were

2 hypofluorescent. The lesion's masking effect was visible at the level of OCT-A

3 (choriocapillaris) with capillary rarefaction of the superficial plexus and the deep plexus

4 (Figure 8). The advanced BSCR lesions that are atrophic with FA colocalized with areas of

5 flow reduction with OCT-A due to the absence of choriocapillaris beneath the disrupted RPE.

6 BSCR is known to cause lesions that might evolve to atrophy of the stroma and the overlying 7 choriocapillaris unless promptly treated.

8 OCT-A performed in the patient with atrophic BC lesions showed blood flow impairment at

9 the level of the choriocapillaris below retinal pigment epithelium disruption (Figure 8).

10

\section{Discussion}

We confirm the pathophysiology of APMPPE, i.e., it is a "primarily choroidal inflammatory disorder leading to outer retinal involvement" because en-face OCT scans at the level of the choriocapillaris in APMPPE showed choroidal infiltration and dilation of choroidal vessels. ${ }^{18}$ Other authors have shown evidence of inner choroidal or choriocapillaris flow reduction or ischemia defects in APMPPE lesions. ${ }^{19,20}$ For instance, those anomalies were demonstrated in $96 \%$ of the eyes with APMPPE for Klufas et al. ${ }^{14}$ In addition, we showed that the hypoperfusion areas of the choriocapillaris regressed with time in a patient with APMPPE, along with his clinical improvement. Similar disappearance of the initial lesions at the level of choriocapillaris have been shown by Burke et al. in a series of 10 eyes. ${ }^{19,20}$

From a pathophysiological viewpoint, APMPPE is a true choroidopathy, whereas MEWDS is instead an epitheliopathy with RPE involvement. ${ }^{18}$ This explains why there is only hypofluorescence in the late frames of the ICGA given the lack of ICG uptake by RPE cells, visible on the late phase of ICGA as suggested by Gaudric and Mrejen and Pichi et al. ${ }^{18,21}$ However, the early phases are normal because there is no choriocapillaris involvement. ${ }^{5}$ There was no choroidal filling defect. Several authors showed completely normal choriocapillaris flow in eyes with MEWDS, with no vessel dilation even in the hyporeflective areas seen in en-face OCT, thus supporting the hypothesis that the choriocapillaris may not be involved in this disease. ${ }^{21,22,23}$ We also confirm their findings that OCT-A analysis of the superficial and deep retinal capillary plexuses was within normal limits.

These observations have already been mentioned in the literature. ${ }^{7}$ Pichi et al. suggest that MEWDS primarily results from inflammation at the RPE and outer photoreceptor level 
1 leading to "photoreceptoritis" causing loss of the inner and outer segments ${ }^{21}$ and

2 inflammation of the photoreceptor segments, with disarray of the ellipsoid. Involvement of the outer retina's microstructures has been described in SD-OCT ${ }^{24,25}$ associated with thicker choroid?. These hyporeflective "spots" on en-face OCT correspond to the classical yellowwhite lesions identified with fundus photography. ${ }^{21}$ Moreover, we confirmed the findings of Pichi et al on imaging of MEWDS. The hypofluorescent lesions in the late frames of ICGA are superimposed on hyporeflective lesions on en-face OCT centered on the ellipsoid and corresponding to ellipsoid disruption areas on SD-OCT. ${ }^{21}$

10 On en-face OCT, the ONL showed tiny hyper-reflective dots corresponding to the punctate hyperreflective material seen in the ONL on SD-OCT. ${ }^{21}$

OCT-A can therefore act as an instrument to differentiate APMPPE and MEWDS, by looking for involvement of the choriocapillaris in APMPPE alone.

In TB-related SLC, our OCTA images showed that choroidal inflammation results in areas of possible flow void at the level of the choriocapillaris. Mandadi et al. hypothesized that these areas may represent either capillary loss/hypoperfusion, or sluggish flow that may be below the limits of detection by OCT-A. ${ }^{22}$ They also concluded that OCTA might be useful in detection of progression of tubercular serpiginous-like choroiditis lesions. ${ }^{26}$

Antituberculous treatment is required in $\mathrm{SLC}^{27}$, and therefore tuberculosis must be sought with clinical manifestations mimicking serpiginous choroiditis. Classically, serpiginous-like choroiditis presents on FA as a hypofluorescence with late hyperfluorescence due to RPE window defects. ICGA showed early and late hypocyanescence with fuzzy margins suggestive of activity.

SD-OCT found outer retinal hyper-reflective deposits with knob-like elevations of RPE;

27 ellipsoid and ELM disruption, atrophy of RPE-Bruch's complex, increased choroidal 28 reflectance, choroidal thinning, and loss of choriocapillaris.

Choroid ischemia with loss of the choriocapillaris and choroidal vessel rarefaction has been 31 suggested as one of the pathophysiological mechanisms of serpiginous choroiditis. Another hypothesis advanced for these abnormalities visualized on OCT-A would be inflammatory 
1 edema or that high-speed/low-speed flow can make visualization of the vascular networks

2 more difficult. ${ }^{28}$

3 Khan and Shahzad's observations on OCT-A further support the role of choriocapillaris loss

4 and hypoperfusion as a contributing factor towards the development of choroidal

5 neovascularization in the later course of resolved serpiginous choroiditis. ${ }^{29}$

6 Therefore, in serpiginous choroiditis, lack of decorrelation signal in OCT-A is probably due to

7 choriocapillaris non perfusion. It is unknown whether this lack of perfusion is due to the

8 obstruction of the capillary lumen or by a compression of capillaries by surrounding

9 inflammation. It is noteworthy that the inner choroid did not appear thinned in areas of 10 serpiginous lesion.

11 We hypothesize that the lesions at the level of the choriocapillaris may correspond to 12 infiltrates of inflammatory cells that obliterate or compress the choriocapillaris. A way to 13 answer this question of hypoperfusion on OCT-A would be to be able to follow the evolution 14 of the choriocapillaris lesions on OCT-A after the inflammatory process has resolved. 15 Unfortunately, we had no follow-up on OCT-A for these cases in our study and we are 16 concerned that the RPE lesions would obscure the analysis of OCT-A at the level of pigment 17 clumps with posterior shadowing (one of our coauthors made that hypothesis in a previous 18 publication $\left.{ }^{30}\right)$.

19 Those hypoperfused area on OCT-A have been shown to correspond topographically to 20 hypofluorescent areas visible on ICGA, although in a case report, El Ameen stated that ICGA 21 remains preferable because it more clearly delineates choriocapillaris lesions. ${ }^{17}$

22 OCT-A showed no decorrelation signal in active lesions in choriocapillaris and the whole 23 choroid. Vessel density of the outer border of inactive lesions seemed to be lower than 24 vessel density of unaffected areas. ${ }^{28}$ Pakzad-Vaezi et al. showed larger lesions on OCT-A in 25 the choriocapillaris during active disease than in the outer nuclear layer and FAF areas. 26 Resolution of those lesions occurred, where OCTA findings were not associated with 27 corresponding abnormal FAF after treatment. Those findings also to support the theory that 28 the choriocapillaris is the primary site of pathology. ${ }^{31}$

29 Of note, one case report showed those areas of "decreased vascularity" in the 30 choriocapillaris, photoreceptor/RPE defects remaining persistent on OCT-A following 31 systemic corticosteroid therapy. ${ }^{32}$ 
1 In both patients with MFC with no associated CNV, OCT-A showed hypoperfusion areas on

2 the choriocapillaris. At resolution, final OCT-A was either stable or showed an hypoperfusion area appearing less extensive than before steroid treatment.

4 Previous studies showed that in active lesions, OCT-A indicates the presence of a small highly organized dense high-flow neovascular network in the outer retina also described as "a collection of capillaries with crippled whitening" ${ }^{8,33}$ Inactive lesions on OCT-A, present with lack of flow in the outer retina and the choriocapillaris.$^{8}$

In patients with CNV associated with MFC and PIC, OCT-A showed flow signal in the morphology of a CNV lesion at the outer retina and the choriocapillaris, as shown previously ${ }^{8,34}$ with reduced flow signal in the immediately adjacent choriocapillaris.

The contribution of OCT-A in the neovascularization associated with MFC has been described in the literature. ${ }^{35}$ OCT-A might be particularly useful in CNV secondary to posterior uveitis known to be distinct from the classic appearance of active CNV on multimodal imaging. Intra-retinal and subretinal fluids on OCT can be missing in the setting of inflammatory CNV like in one eye in our series (as shown in figure $11^{\prime}$ ) and fluorescein angiography often present a mixed staining and leakage from inflammatory lesions. ${ }^{9}$ Therefore, OCT-A could be helpful in distinguishing CNV from inflammatory lesions keeping in mind that in some recurrent cases OCTA cannot not reliably give information about CNV activity (in contrast to FA) and thus, FA might still necessary to make therapy decisions in such cases. ${ }^{36}$ Based on preexisting OCT-A stratification of different CNV patterns/shapes, Pohlman et al. have described the ones associated with active PIC diseases as 'lacy wheel shape', 'pruned large-trunk vessels' or just hypoperfusion with one vessel formation, while most stable eyes presented with 'dead tree aspect' vessels. Moreover, 'lacy wheel shape' and 'pruned largetrunk' vessels were found above the RPE, whereas 'dead tree aspect' vessels were observed below the RPE. ${ }^{33}$ For Zahid et al, although OCTA seems sensitive for detecting neovascular 27 flow within MFC lesions, it seems to have low specificity in determining which lesions are clinically active. Indeed, they showed that neovascular flow persists in the large majority of MFC lesions, including those that appear inactive on clinical examination and most other imaging modalities. They detected neovascular flow in $83 \%$ of the macular chorioretinal active and inactive MFC lesions (subretinal, and mixed) evaluated, while purely sub-RPE lesions did not demonstrate neovascular flow on OCT-A. This represents a much higher 
1 frequency than that reported by other authors using different imaging methods. Of the

2 subretinal lesions that were judged to be inactive based on clinical examination, FA and

3 structural OCT, they found that $88 \%$ showed neovascular flow when analyzed with OCTA.

4 They concluded that the FA patterns of inactive MFC lesions containing fibrovascular tissue

5 might not be interpreted as representing NV when there is no angiographic leakage. ${ }^{34}$

7 De Carlo et al. have hypothesized that the areas of perceived choriocapillaris "loss" in the 8 birdshot lesions may represent either true vessel atrophy or, alternatively greatly reduced 9 blood flow in these regions. ${ }^{37}$ We also confirmed that OCT angiograms demonstrated that 10 larger choroidal vessels bordered the atrophic birdshot lesion. De Carlo et al. also speculated 11 about whether these larger vessels represent a compensatory response by the choroid or if 12 they are simply vessels from Sattler's layer being pushed into an atrophic choriocapillaris 13 plane.

14 On contrary, acute choroidal lesions in BSCR, are composed of infiltrates of epithelioid cells, 15 and therefore they are not visible on OCT-A because not showing alterations of the 16 choriocapillaris flow and OCT-A only visualizes the flow (movements of the cellular blood 17 components). 4,38 However, the patient in our series who presented a recent diagnosis of 18 birdshot, showed only choroid flow voids by OCT-A and hypofluorescent dots on ICG without 19 any white dots on color pictures nor choriocapillaris alteration (Figure 10').

20 Pohlman et al. have analyzed the OCT-A findings in 64 eyes with BSCR and they described 21 capillary loops in 58\%, telangiectatic vessels in 44\%, increased intercapillary spaces in $52 \%$, 22 and altered vascular architecture in $53 \%$ of eyes in superficial capillary plexus (SCP). 23 Interestingly, the authors observed non-perfused areas with a decreased number of vessels 24 in all retinal layers; however, they were not visible in deeper layers. In total, a rarefication of 25 C-scans (or en face images derived from the B-scans) was seen in 63\% of eyes in SCP and in $2652 \%$ of eyes in DCP in their study. There found no significant differences in capillary loops, 27 telangiectatic vessels, or increased intercapillary spaces whatever was the stage of the 28 disease was, recent active to advanced inactive stages. However, the alteration of vascular 29 architecture and the increased rarefication were increased with time of duration of 30 disease. ${ }^{39}$ For de Carlo et al. the most noticeable change in the retinal vasculature was an 31 increased intercapillary space in all eyes, $75 \%$ in their study. ${ }^{37}$ We also noticed this change 32 in both eyes along with telangiectatic vessels in our patient with 12 years history of 
advanced, yet inactive, birdshot disease and capillary loop in both of our patients with recent and old BSCR disease (Figures 9' and 10').

The main limitation of our study is the low number of subjects for each pathology because the study was conducted in specialized uveitis centers from referrals. Nonetheless, we strongly believe that the study provides a thorough description of a number of cases which might be grouped as "white dot syndromes," by OCT-A. This report is strengthened by the fact that we have extensive retinal imaging, including OCT-A, color pictures, autofluorescence, fluorescein angiography and ICGA.

We found that OCT-A allows to distinguish 2 types of inflammatory disorders. The one involving primarily the choriocapillaris including serpiginous choroiditis, tuberculosis (TB) related SLC, and APMPPE. And the one involving the outer retina and RPE including MEWDS that can spontaneously heal without sequelae.

In APMPPE, OCT-A shows 2 different classes of lesions: 1 / some lesions appear as hypoperfusion of the choriocapillaris on OCT-A showing up as scars on SD-OCT during progression, 2/ the other lesions show up as hyporeflective on OCT-A, and are potentially reversible.

Comparing the OCT-A features of those entities allowed us to suggest that both entities APMPPE and SLC might be part of the same spectrum of inflammatory disease with primary involvement of the level of the choriocapillaris and secondary RPE damage, which is a new finding. In AMPPE, the lesions are often serpiginous in their distribution, though.

In summary, to be able to assess an involvement of the RPE without hypoperfusion of the choriocapillaris, the following are needed: no hypofluorescence on early FA and late FA, no hypofluorescence on early ICGA but presence of a hypofluorescence on late ICGA (at 30 minutes). If OCT-A is contradictory with the result of ICGA as regard as a flow void in the choriocapillary, OCT-A need to be analyzed keeping in mind that an opacification of EPR can give a shadowing effect on the choriocapillaris. 
1 Finally, OCT-A contributes pathophysiological information in "white dot syndromes" and

2 choroiditis and can detect hypoperfusion of the choriocapillaris as well as associated CNV.

3 OCT-A can therefore be integrated into multimodal imaging of these diseases.

4

5

6

7

8

9

10

11

12

13

14

15

16

17

18

19

20

21

22

23

24

25

26

27

28

29

30

31

32

33

34

35

36

\section{References}

1. Schaal S, Schiff W, Kaplan H, Tezel T. Simultaneous appearance of multiple evanescent white dot syndrome and multifocal choroiditis indicate a common causal relationship. Ocul Immunol Inflamm. 2009;17(5):325-7.

2. Zweifel S, Kim A, Freund B. Simultaneous presentation of multifocal choroiditis and acute zonal occult outer retinopathy in one eye. Br J Ophthalmol. 2011;95(2):288, 297-8.

3. Bryan R, Freund K, Yannuzzi L, Spaide R, Huang S, Costa D. Multiple evanescent white dot syndrome in patients with multifocal choroiditis. Retina. 2002;22(3):317-22.

4. Pichi F, Sarraf D, Arepalli S, et al. The application of optical coherence tomography angiography in uveitis and inflammatory eye diseases. Prog Retin Eye Res. 2017;59:178201.

5. Mangeon M, Zett C, Amaral C, Novais E, Muccioli C, Andrade G, et al. Multimodal Evaluation of Patients with Acute Posterior Multifocal Placoid Pigment Epitheliopathy and Serpiginous Choroiditis. Ocul Immunol Inflamm. 2017;1-7.

6. Heiferman MJ, Rahmani S, Jampol LM, et al. Acute posterior multifocal placoid pigment epitheliopathy on optical coherance tomography angiography. Retina. 2017;37(11):2084 $-94$.

7. De Bats F, Wolff B, Vasseur V, et al. « En-face » spectral-domain optical coherence tomography findings in multiple evanescent white dot syndrome. J Ophthalmol. 2014;2014:928028.

8. Astroz P, Miere A, Mrejen S, et al. Optical coherence tomography angiography to distinguish choroidal neovascularization from macular inflammatory lesions in multifocal choroiditis. Retina. 2018;38(2):299-309.

9. Levison AL, Baynes KM, Lowder CY, Kaiser PK, Srivastava SK. Choroidal neovascularisation on optical coherence tomography angiography in punctate inner choroidopathy and multifocal choroiditis. Br J Ophthalmol. 2017;101(5):616-622. 
10. Zahid S, Chen KC, Jung JJ, Balaratnasingam C, Ghadiali Q, Soreson J, et al. Optical coherence tomography angiography of chorioretinal lesions due to idiopathic multifocal choroiditis. Retina. 2017;37(8):1451-1463

11. Astroz P, Miere A, Mrejen S, Sekfali R, Souied EH, Jung C, et al. Optical coherence tomography angiography to distinguish choroidal neovascularization from macular inflammatory lesions in multifocal choroiditis. Retina. 2018;38(2):299-309.

12. Spaide RF, Koizumi H, Pozzoni MC, Pozonni MC. Enhanced depth imaging spectraldomain optical coherence tomography. Am J Ophthalmol. 2008;146(4):496-500.

13. Rosenfeld PJ, Durbin MK, Roisman L, et al. ZEISS Angioplex ${ }^{\mathrm{TM}}$ Spectral Domain Optical Coherence Tomography Angiography: Technical Aspects. Dev Ophthalmol. 2016;56:18-29.

14. Klufas MA, Phasukkijwatana N, lafe NA, Prasad PS, Agarwal A, Gupta V, et al. Optical coherence tomography angiography reveals choriocapillaris flow reduction in placoid chorioretinitis. Ophthalmol Retina 2017:1: 77-91.

15. Agarwal A, Aggarwal K, Deokar A, et al. Optical Coherence Tomography Angiography Features of Paradoxical Worsening in Tubercular Multifocal Serpiginoid Choroiditis. Ocul Immunol Inflamm. 2016;24(6):621-30.

16. Wang H, Tan SZ, Aslam T, Jones NP, Steeples LR. Multimodal Evaluation of Presumed Tuberculous Serpiginous-Like Choroiditis. Ocul Immunol Inflamm. 2018;1-5.

17. El Ameen A, Herbort CP. serpiginous choroiditis imaged by optical coherence tomography angiography. Retin Cases Brief Rep. 2016;12(4):279-28516.

18. Gaudric A, Mrejen S. why the dots are black only in the late phase of the indocyanine green angiography in multiple evanescent white dot syndrome. Retin Cases Brief Rep. 2017;11 Suppl 1:S81-5.

19. Burke TR, Chu CJ, Salvatore S, Bailey C, Dick AD, Lee RWJ, et al. Application of OCTangiography to characterize the evolution of chorioretinal lesions in acute posterior multifocal placoid pigment epitheliopathy. Eye. 2017;31, 1399-1408

20. Werner JU, Enders C, Lang GK, Lang GE. Multi-Modal Imaging Including Optical Coherence Tomography Angiography in Patients With Posterior Multifocal Placoid Pigment Epitheliopathy. Ophthalmic Surg Lasers Imaging Retina. 2017;48(9):727-733.

21. Pichi F, Srvivastava SK, Chexal S, et al. En face optical coherence tomography and optical coherence tomography angiography of multiple evanescent white dot syndrome: New Insights Into Pathogenesis. Retina. 2016;36 Suppl 1:S178-88. 
1

2

3

4

5

6

7

8

9

10

11

12

13

14

15

16

17

18

19

20

21

22

23

24

25

26

27

28

29

30

31

32

33

34

35

36

37

38

39

40

41

42

43

44

45

22. Yannuzzi NA, Miller A, Gregori G, Davis JL, Rosenfeld PJ. Swept-source

OCT angiography shows sparing of the choriocapillaris in multiple evanescent white dot syndrome. Ophthalmic Surg Lasers Imaging Retina. 2017;48(1):69-74

23. Pereira F, Lima Lh, De Azevedo Agb, Zett C, Farah Me, Belfort R, Jr. Swept-source OCT in patients with multiple evanescent white dot syndrome. J Ophthalmic Inflamm Infect.

2018;8(1):16.

24. Amin HI. Optical coherence tomography findings in multiple evanescent white dot syndrome. Retina 2006;26(4):483-484.

25. Sikorski BL, Wojtkowski M, Kaluzny JJ, Szkulmowski M, Kowalczyk A. Correlation of spectral optical coherence tomography with fluorescein and indocyanine green angiography in multiple evanescent white dot syndrome. Br J Ophthalmol 2008;92(11):1552-1557.

26. Mandadi SKR, Agarwal A, Aggarwal K, et al; for OCTA Study Group. novel findings on optical coherence tomography angiography in patients with tubercular serpiginous-like choroiditis. Retina. 2017;37(9):1647-1659.

27. Bansal R, Gupta A, Gupta V, Dogra MR, Sharma A, Bambery P. Tubercular serpiginouslike choroiditis presenting as multifocal serpiginoid choroiditis. Ophthalmology. 2012;119(11):2334-42.

28. Montorio D, Giuffrè C, Miserocchi E, et al. Swept-source optical coherence tomography angiography in serpiginous choroiditis. Br J Ophthalmol. 2018;102(7):991-995.

29. Khan HA, Shahzad MA. Multimodal Imaging of Serpiginous Choroiditis. Optom Vis Sci. 2017;94(2):265-269.

30. Mrejen S, Sarraf D, Chexal S, Wald K, Freund KB. Choroidal Involvement in Acute Posterior Multifocal Placoid Pigment Epitheliopathy. Ophthalmic Surg Lasers Imaging Retina. 2016;47(1):20-6.

31. Pakzad-Vaezi K, Khaksari K, Chu Z, Van Gelder RN, Wang RK, Pepple Kl. Swept-source OCT angiography of serpiginous choroiditis. Ophthalmol Retina. 2018;2(7):712-9.28.

32. Ahn SJ, Park SH, Lee BR. Multimodal Imaging Including Optical Coherence Tomography Angiography in Serpiginous Choroiditis. Ocul Immunol Inflamm. 2017;25(2):287-291.

33. PohImann D, Pleyer U, Joussen AM, Winterhalter S. Optical coherence tomography angiography in comparison with other multimodal imaging techniques in punctate inner choroidopathy. Br J Ophthalmol 2019;103:60-66

34. Zahid S, Chen KC, Jung JJ, et al. optical coherence tomography angiography of chorioretinal lesions due to idiopathic multifocal choroiditis. Retina. 2017;37(8):145163. 
35. Baumal CR, de Carlo TE, Waheed NK, Salz DA, Witkin AJ, Duker JS. Sequential Optical Coherence Tomographic Angiography for Diagnosis and Treatment of Choroidal Neovascularization in Multifocal Choroiditis. JAMA Ophthalmol. 2015;133(9):1087-90.

36. Cheng L, Chen X, Weng S, Mao L, Gong Y, Yu S, et al. Spectral-domain optical coherence tomography angiography findings in multifocal choroiditis with active lesions. Am J Ophthalmol. 2016;169:145-61.

37. De Carlo TE, Bonini Filho MA, Adhi M, Duker JS. Retinal and choroidal vasculature in birdshot chorioretinopathy analyzed using spectral domain optical coherence tomography angiography. Retina. 2015;35(11):2392-9.

48. Invernizzi A, Cozzi M, Staurenghi G. Optical Coherence Tomography and Optical Coherence Tomography Angiography in Uveitis: A Review. Clin Exp Ophthalmol. 2019;47(3):357-371.

39. Pohlmann D, Macedo S, Stübiger N, Pleyer U, Joussen AM, Winterhalter S. Multimodal Imaging in Birdshot Retinochoroiditis. Ocul Immunol Inflamm. 2017;25(5): 621-632

\section{Acknowledgments}

The authors thank Mrs Linda Northrup for her assistance in translation and Mr. Chad Indermill from the Imaging Department of Ophthalmology at UPMC, Pittsburgh, PA, USA.

Figure 1. Multimodal imaging of a patient with APMPPE (Patient 1): (A) On fundus FAF, active lesions are mostly hyperautofluorescent. (B) Early phase of FA showed choriocapillaris hypoperfusion with a similar pattern. (C) The border of those lesions appeared hyperfluorescent at later phase. (D) OCT-A revealed areas of hypoperfusion of the choriocapillaris on OCT-A, which was superimposed on the hypofluorescent areas in the early frames of ICGA $(E, F, G)$. (H) SD-OCT showed areas of IS-OS layer disruption and thickened areas as well as outer retinal hyperreflectivity.

FAF: autofluorescence; IS-OS: Inner segment-outer segments

Figure 2. OCT Angiography of two patients with APMPPE: $(A, B, C, D)$ OCT-A showed no flow 
1 abnormalities at the superficial and deep plexuses of the two patients with APMPPE. (G, H)

2 They presented areas of hypoperfusion of the choriocapillaris on OCT-A, visible as a circulatory defect on the choriocapillary plane (yellow stars), which was superimposed on the hypofluorescent areas in the early frames of ICGA $(\mathrm{I}, \mathrm{J})$.

(E, F) Outer retina on OCT-A.

Figure 3. Multimodal imaging of a patient with MEWDS (Patient 3): $(A, B, C, D, E)$ The lesions appear hyperautofluorescent, hyperfluorescent on the late frames of FA and hypofluorescent on the late frames of ICG. (G) SD-OCT showed disruption of ellipsoid and interdigidation zone, meaning disruption of photoreceptors. (H) En-face OCT showed hyperreflective dots in the ONL. (I) En-face OCT centered on the ellipsoid zone showed hyporeflective areas corresponding to IS/OS layer disruption and were superimposed on hypofluorescent lesions visible in the late frames of ICGA (F). FAF: autofluorescence; IS/OS: Inner segment-outer segment; ONL: outer nuclear layer

Figure 4. OCT Angiography of two patients with MEWDS (Patients 3 \& 4): $(A, B, C, D, E, F)$ OCT-A showed no flow abnormalities at superficial $(A, B)$, deep capillary plexus (C,D), outer retina $(E, F)$ and $(G, H)$ at choriocapillaris levels.

\section{Figure 5. Multimodal imaging of a patient with presumed TB-related serpiginous-like} choroiditis (Patient 6): (A) On initial FAF, the lesions presented as hypoautofluorescent surrounded by an hyperautofluorescent border. (B,C) FA showed hyperfluorescence at intermediate frames and increased in the later frames. $(E, F)$ ICGA showed confluent hypofluorescent lesions in the early and late frames. (D) OCT-A showed hypoperfusion areas of the choriocapillaris larger than the lesions at the level of the RPE on FA. Although some hypointensities were reversible on FAF after appropriate treatment (shown as yellow stars on OCT-A), other lesions failed to recover (surrounded in a red circle on OCT-A). (G) Superimposition between the choriocapillaris hypoperfusion lesions on OCT-A and the hyperfluorescent lesions on ICG were noted. (H) SD-OCT demonstrated areas of IS/OS layer disruption and areas of hyperreflectivity in the outer nuclear layer (arrows), atrophy of the outer retina with cysts, and hyper-reflectivity of the 
1 opposite choroid. (I). On final FAF after treatment, the lesion failed to recover showed up as

2 hypoautofluorescent (red circle), whereas the other lesions were iso or

3 hyperautofluorescent (yellow circles).

4 FAF: autofluorescence; RPE: Retinal Pigment Epithelium; IS/OS: Inner segment-outer

5 segments

6

7

Figure 6. Multimodal imaging of a patient with serpiginous choroiditis (Patient 7): (A) FA

showed hypofluorescent confluent lesions in the early frames, which became

9

10 hyperfluorescent on the late frames (C) with an incomplete hyperfluorescent edges indicating the disease activity. (B,D) ICGA showed these same lesions as remaining hypofluorescent throughout the sequence. (E) Fundus colour picture showing the yellowish lesions. (F) OCT-A showed hypoperfusion lesions of the choriocapillaris that superimposed with the hyperfluorescent lesions in the late frame of FA.

Figure 7. Patients 8, 9, 10 with MFC: In eyes of the two patients (patients 9: 'MFC 1' and 11: 'MCF 2') without CNV, (J,K) OCT-A showed hypoperfusion areas on the choriocapillaris superimposed on hypofluorescence lesions on ICGA (M,N).

In the eye of patient 10 ('MFC with neovascularization'), OCT-A showed the CNV at the outer retina (I) as a hyper-reflective glomerule with a choriocapillary hyperreflective area (L). The superimposed ICGA showed an hypofluorescent lesion (O).

$(A, B, C, D, E, F)$ OCT-A showed normal superficial and deep capillary plexuses.

MFC: multifocal choroiditis

Figure 8. Patient 16 with birdshot chorioretinopathy :

ICGA showed widespread hypofluorescence dots in the left eye (A). En face OCT at the level of the IS/OS Ellipsoid showed decreased hyperreflectivity at the fovea (B). Foveal OCT-A $6 \times$ $6 \mathrm{~mm}$ of retinal vasculature showed changes in deep capillary plexus (DCP) showing capillary loops (white arrows), OCT-A does no showed choriocapillaris alteration in maculae (D) and choroid flow voids at the birdshot lesion inferior to the fovea and to the optic nerve (orange 
1 arrows (E). The SD-OCT scans shows diffuse slight reduction of the reflectivity of both the EZ

2 and the IZ (area between arrows), although both are intact.

3 IS/OS: inner segment/outer segment junction

4

5

6

7

\section{Supplemental Material :}

Figure 2' OCT Angiography evolution of the lesions in patient with APMPPE (Patient 1).

Most lesions are hyperautofluorescent or isofluorescent and some are hypoautofluorescent (red circle) at initial FAF. The hyperautofluorescent and isofluorescent lesions seen on FAF correspond to the lesions that fade away on OCT-A over time. B,C,D. OCT-A at the level of the choriocapillaris. During 2 months of follow-up, two different patterns of active lesions were detected. Although lesions at the level of the choriocapillaris (yellow circles) are reversible, the lesion that fails to recover on OCT-A (red circle) appears hypoautofluorescent initially and was located at the level of RPE and choriocapillaris. E. At final FAF, hypofluorescent lesions are smaller than at initial FAF but persistent. The persistent lesion on OCT-A (red circle) does not show up on final FAF. F,G,H. En face OCT at the level of choriocapillaris/ choroid. Lesions at the level of the choriocapillaris/ choroid (yellow circles) are reversible, the lesions that fails to recover (red circle) appear hyperreflective. APMPPE: Acute posterior multifocal placoid pigment epitheliopathy

\section{Figure 4'. OCT Angiography of one patient with MEWDS (Patient 5): (A-K)} OCT-A showed no flow abnormalities at superficial $(A, B)$, deep capillary plexus $(C, D)$, outer retina $(E, F)$ and $(G, H)$ at choriocapillaris levels.

\section{Figure 6'. SD-OCT and OCT Angiography of a patient with serpiginous choroiditis (Patient} 7): $(A, B, C)$ OCT-A showed no flow abnormalities of the superficial $(A)$ and deep capillary plexuses (B) and the outer retina (C). (D) There were flow impairment at the level of the choriocapillaris (yellow and red circles). Larger choroidal vessels have been pushed inward into the area of choriocapillaris alteration so are seen (red circles on OCT-A). (F) Corresponding SD-OCT scan shows the loss of RPE causing increased intensity below Bruch's membrane which is characteristic RPE atrophy. (F) SD-OCT found areas with atrophy of the outer retina and ellipsoid disruption with retinal cysts regarding RPE atrophy (yellow arrow). 
1 (E) These lesions associated with RPE atrophy appeared hypoautofluorescent on FAF (red

2 circles) although other lesions were rather hyperautofluorescent (yellow circles).

3 FAF: autofluorescence; RPE: Retinal Pigment Epithelium

4

5 Figure 7'. Patient 11 with MFC without CNV : (A) On OCT-A the lesion was less extensive than it appeared on AF (B) and early ICGA (C). (D) The OCT-A images were superimposed on the hypofluorescent areas in the early frames of ICGA. AF : autofluorescence; CNV: chorioretinal neovascularization

Figure 7". Patients 12 (A-G) and 13 (H-N) with MFC and CNV:

OCT-A showed the CNVs at the outer retina $(C, J)$ as hyper-reflective glomerules with a choriocapillary hyperreflective area (red circles). Note also hypoperfusion areas on the choriocapillaris (yellow circles). The fluorescein angiography showed late leakage from the CNVs $(G, N)$ better seen than on the early frame $(F)$. $(A, B, H, I)$ OCT-A showed normal superficial and deep capillary plexuses. SD-OCT showed CNVs with subretinal fluid (E,L). Fundus color picture (patient 13) showed widespread chorioretinal lesions without hemorrhage (M).

MFC: multifocal choroiditis; CNV: chorioretinal neovascularization

Figure 7'"'. Patient 14 (A-B) with PIC and CNV:

21 OCT-A showed the CNV at the choriocapillaris (A, top) as a hyper-reflective glomerule area. On $B$, the CNV is no longer seen one month after anti-VEGF ocular injection. SD-OCT (A) showed CNV without intraretinal or subretinal fluid.

MFC: punctate inner choroiditis; CNV: chorioretinal neovascularization

Figure 9'. Patient 15 with birdshot chorioretinopathy :

En face OCT at the level of the IS/OS ellipsoid superimposed with ICGA (in background) showed decreased hyperreflectivity nasal from the optic nerves in both eyes $(A, B)$ and in the birdshot lesion at the fovea in the left eye (B). OCT-A showed choriocapillaris alteration in 31 both maculae (C,D) and decreased blood flow at the birdshot lesion in the fovea in the left eye (D). The $6 \times 6 \mathrm{~mm}$ OCT-A of the left (D) eye showing larger choroidal vessels bordering 
1 the birdshot lesion (yellow arrow). (G,I) Foveal OCT-A $6 \times 6 \mathrm{~mm}$ of retinal vasculature

2 changes in superficial capillary plexus (SCP) showing telangiectatic vessels (red circles),

3 capillary loops (white arrows), and abnormally increased intercapillary spaces or rather non-

4 perfused areas (blue asterisk). The SD-OCT scans are presented below the OCT-A $(H, J)$ and

5 showed altered reflectivity of both the EZ and the $I Z$.

6 IS/OS: inner segment/outer segment junction

7

8

9 


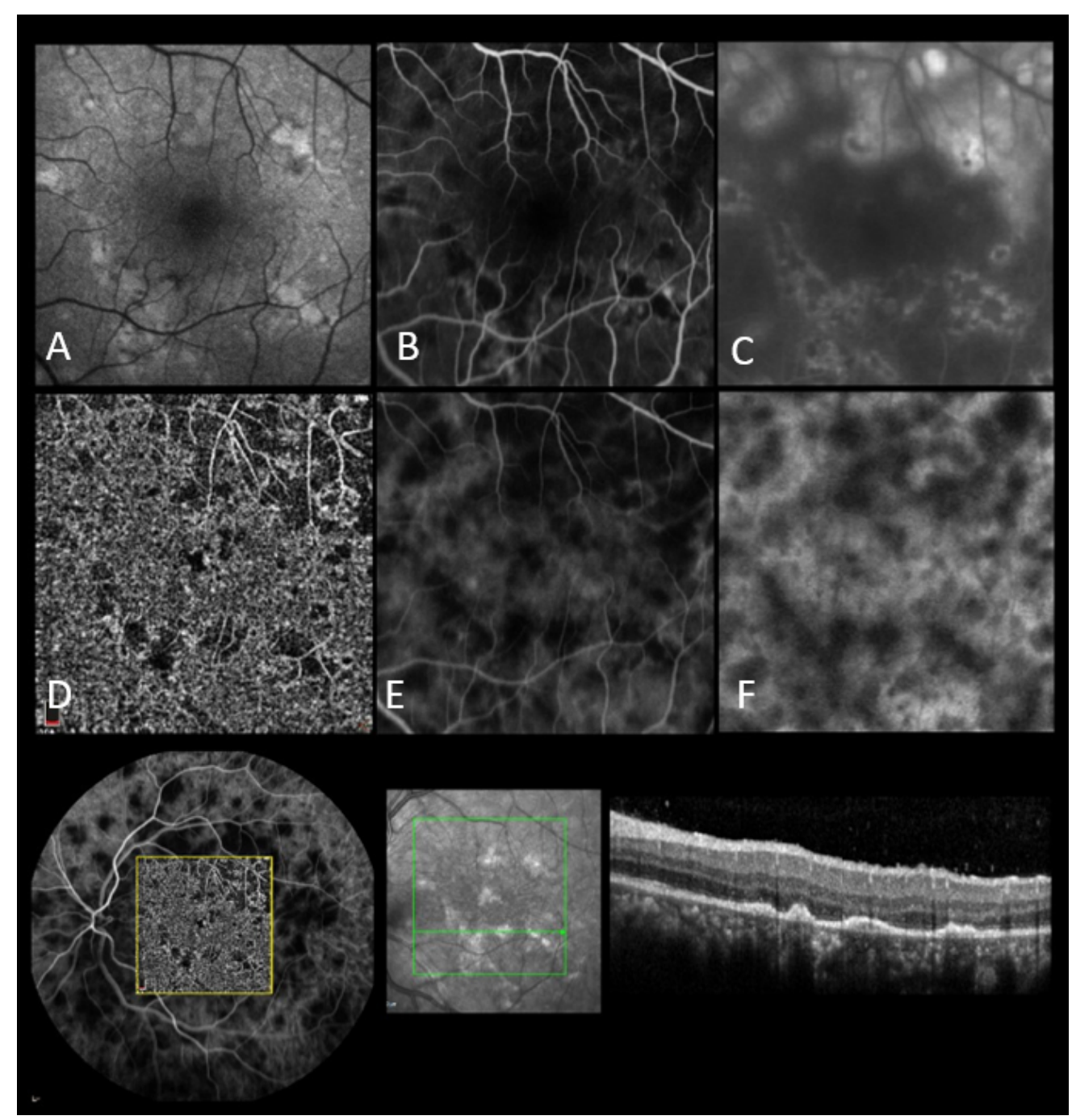

Figure 1. Multimodal imaging of a patient with APMPPE (Patient 1): (A) On fundus FAF, active lesions are mostly hyperautofluorescent. (B) Early phase of FA showed choriocapillaris hypoperfusion with a similar pattern. (C) The border of those lesions appeared hyperfluorescent at later phase. (D) OCT-A revealed areas of hypoperfusion of the choriocapillaris on OCT-A, which was superimposed on the hypofluorescent areas in the early frames of ICGA $(E, F, G)$. (H) SD-OCT showed areas of IS-OS layer disruption and thickened areas as well as outer retinal hyperreflectivity. FAF: autofluorescence; IS-OS: Inner segment-outer segments $115 \times 120 \mathrm{~mm}(150 \times 150 \mathrm{DPI})$ 
Figure 2. OCT Angiography of two patients with APMPPE: $(A, B, C, D)$ OCT-A showed no flow abnormalities at the superficial and deep plexuses of the two patients with APMPPE. (G, H)

They presented areas of hypoperfusion of the choriocapillaris on OCT-A, visible as a circulatory defect on the choriocapillary plane (yellow stars), which was superimposed on the hypofluorescent areas in the early frames of ICGA $(I, J)$. $(E, F)$ Outer retina on OCT-A.

\author{
$51 \times 121 \mathrm{~mm}(150 \times 150 \mathrm{DPI})$
}




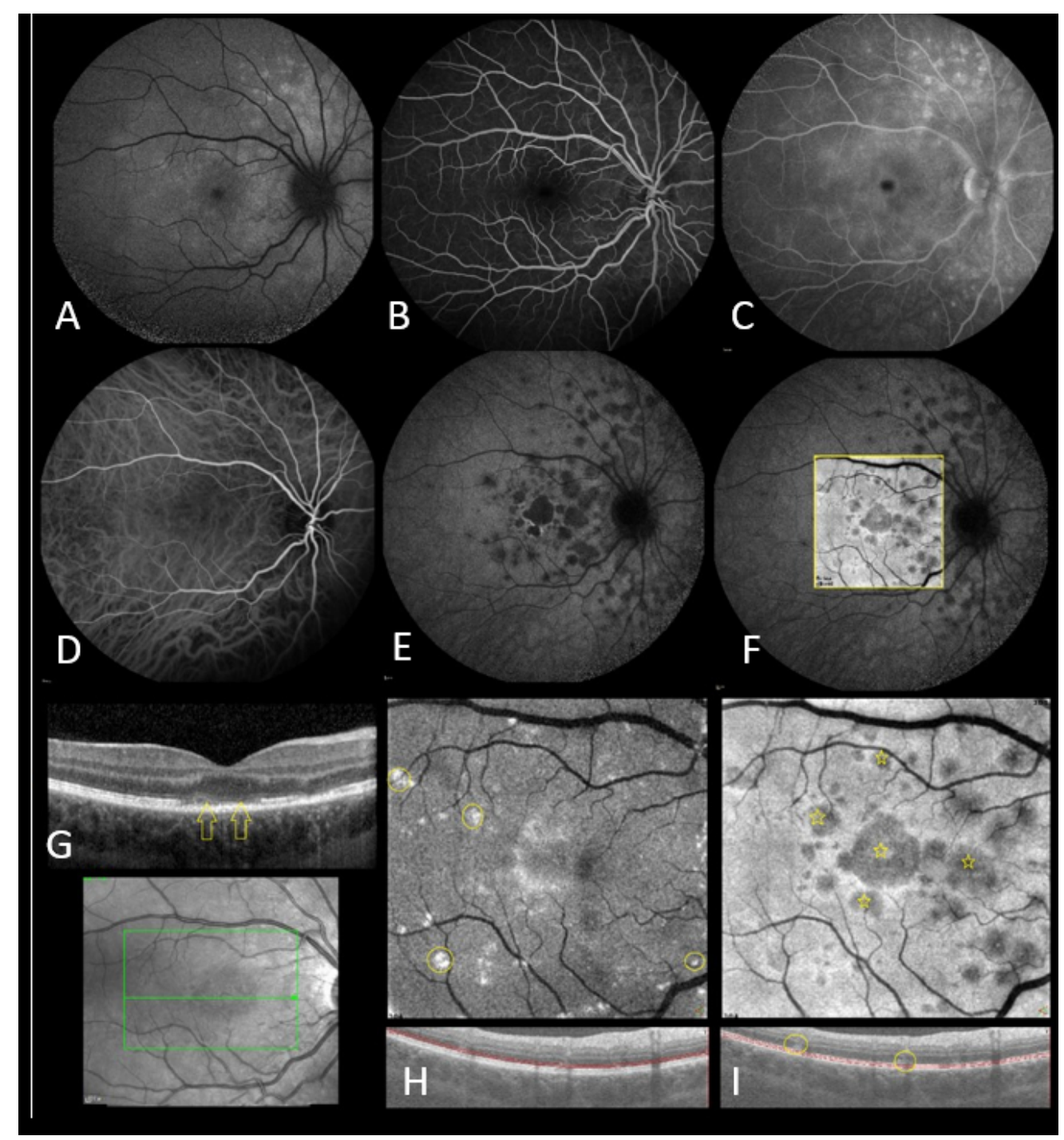

Figure 3. Multimodal imaging of a patient with MEWDS (Patient 3): $(A, B, C, D, E)$ The lesions appear hyperautofluorescent, hyperfluorescent on the late frames of FA and

hypofluorescent on the late frames of ICG. (G) SD-OCT showed disruption of ellipsoid and interdigidation zone, meaning disruption of photoreceptors. $(\mathrm{H})$ En-face OCT showed hyper- reflective dots in the ONL. (I) En-face OCT centered on the ellipsoid zone showed hyporeflective areas corresponding to IS/OS layer disruption and were superimposed on hypofluorescent lesions visible in the late frames of ICGA (F).

FAF: autofluorescence; IS/OS: Inner segment-outer segment; ONL: outer nuclear layer

$113 \times 122 \mathrm{~mm}(150 \times 150 \mathrm{DPI})$ 


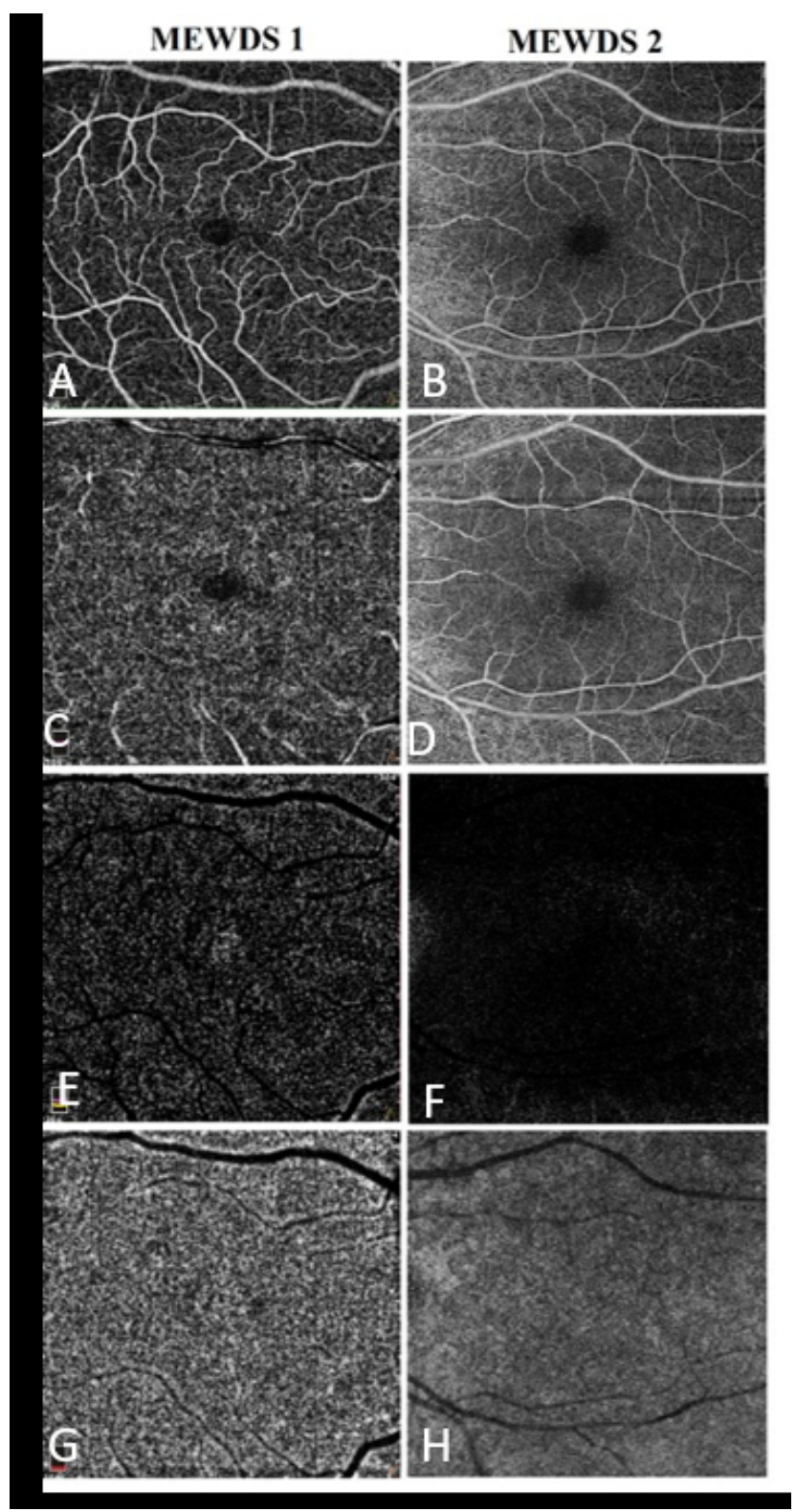

Figure 4. OCT Angiography of two patients with MEWDS (Patients $3 \& 4):(A, B, C, D, E, F)$ OCT-A showed no flow abnormalities at superficial $(A, B)$, deep capillary plexus $(C, D)$, outer retina $(E, F)$ and $(\mathrm{G}, \mathrm{H})$ at choriocapillaris levels.

\author{
$63 \times 122 \mathrm{~mm}(150 \times 150 \mathrm{DPI})$
}




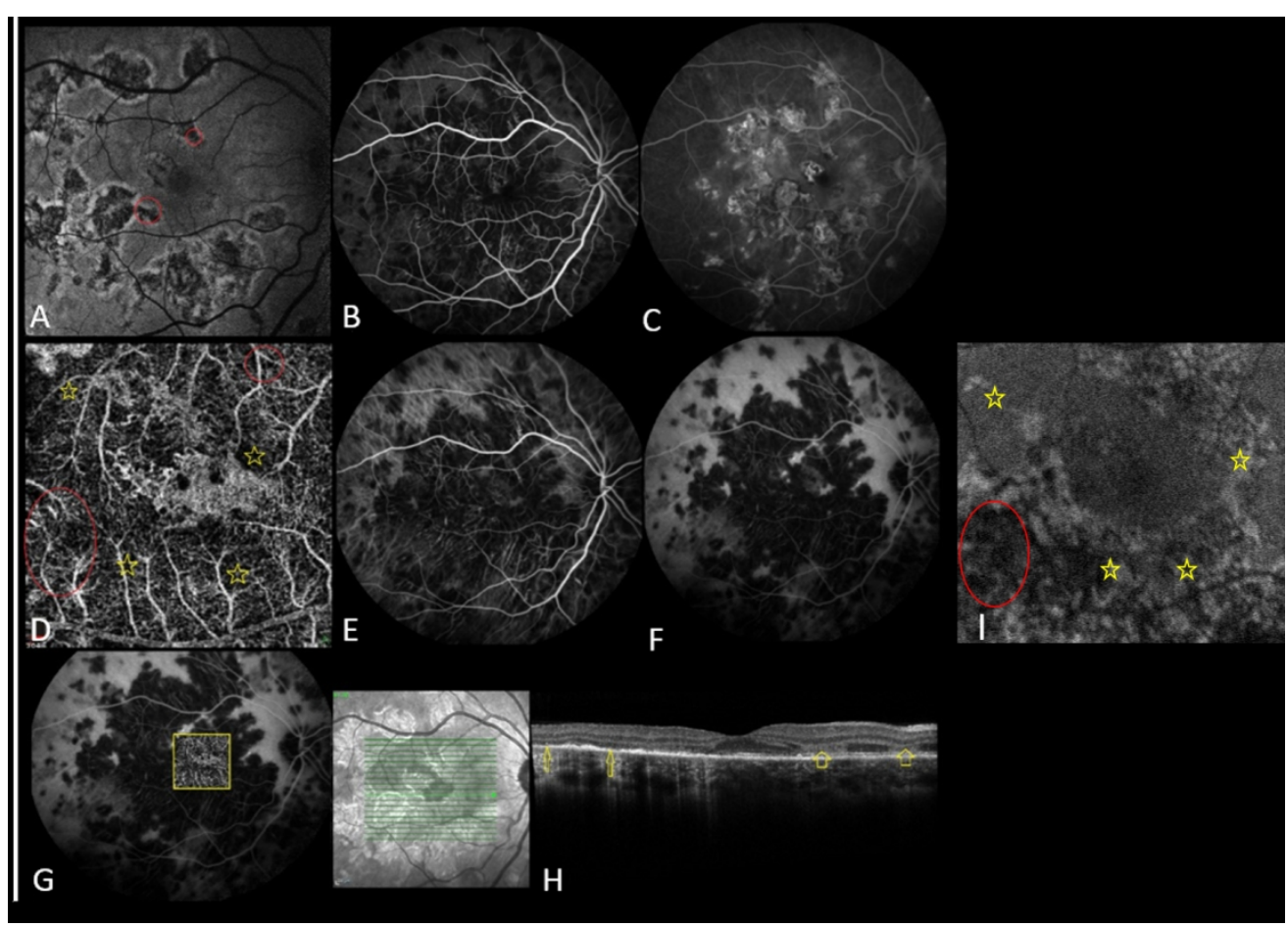

Figure 5. Multimodal imaging of a patient with presumed TB-related serpiginous-like choroiditis (Patient 6): (A) On initial FAF, the lesions presented as hypoautofluorescent surrounded by an hyperautofluorescent border. $(B, C)$ FA showed hyperfluorescence at intermediate frames and increased in the later frames. $(E, F)$

ICGA showed confluent hypofluorescent lesions in the early and late frames. (D) OCT-A showed hypoperfusion areas of the choriocapillaris larger than the lesions at the level of the RPE on FA. Although some hypointensities were reversible on FAF after appropriate treatment (shown as yellow stars on OCT-A), other lesions failed to recover (surrounded in a red circle on OCT-A). (G) Superimposition between the choriocapillaris hypoperfusion lesions on OCT-A and the hyperfluorescent lesions on ICG were noted. (H) SD-OCT demonstrated areas of IS/OS layer disruption and areas of hyperreflectivity in the outer nuclear layer (arrows), atrophy of the outer retina with cysts, and hyper-reflectivity of the opposite choroid. (I). On final FAF after treatment, the lesion failed to recover showed up as hypoautofluorescent (red circle), whereas the other lesions were iso or hyperautofluorescent (yellow circles). FAF: autofluorescence; RPE: Retinal Pigment Epithelium; IS/OS: Inner segment-outer segments

$266 \times 189 \mathrm{~mm}(150 \times 150 \mathrm{DPI})$ 


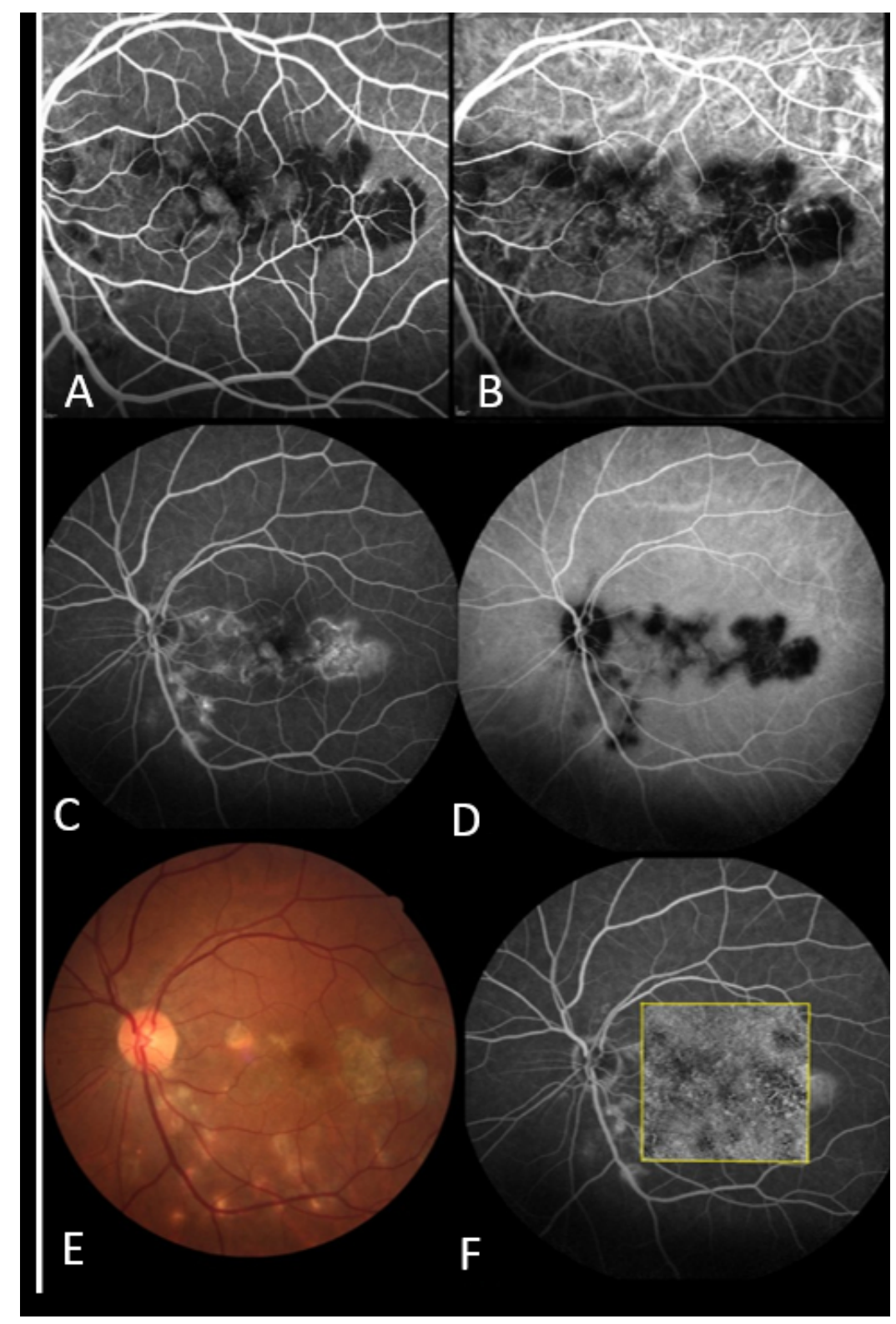

Figure 6. Multimodal imaging of a patient with serpiginous choroiditis (Patient 7): (A) FA showed hypofluorescent confluent lesions in the early frames, which became hyperfluorescent on the late frames (C) with an incomplete hyperfluorescent edges indicating the disease activity. (B,D) ICGA showed these same lesions as remaining hypofluorescent throughout the sequence. (E) Fundus colour picture showing the yellowish lesions. (F) OCTA showed hypoperfusion lesions of the choriocapillaris that superimposed with the hyperfluorescent lesions in the late frame of FA.

$82 \times 123 \mathrm{~mm}(150 \times 150 \mathrm{DPI})$ 


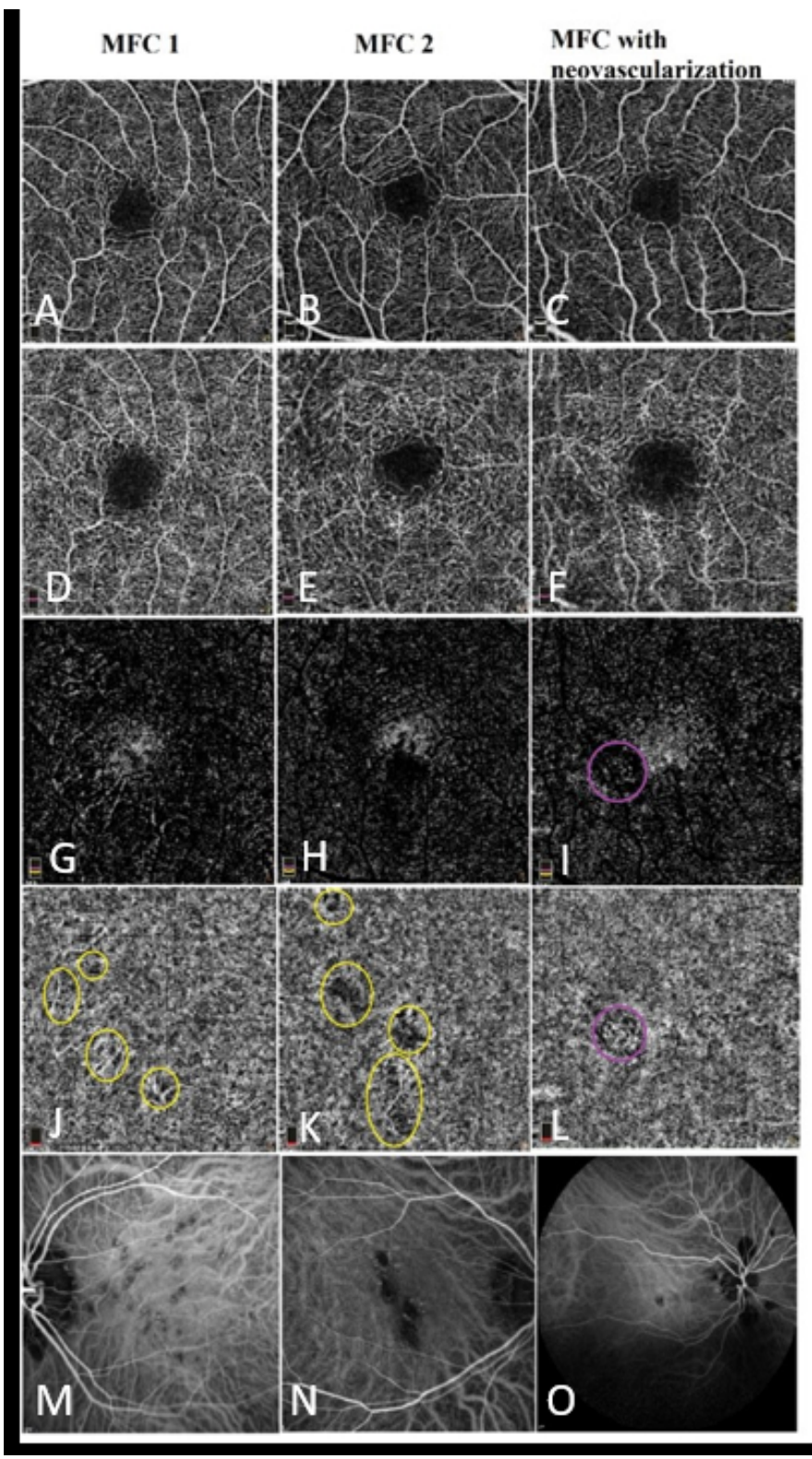

Figure 7. Patients 8, 9, 10 with MFC: In eyes of the two patients (patients 9: 'MFC 1 ' and 11: 'MCF 2') without CNV, (J,K) OCT-A showed hypoperfusion areas on the choriocapillaris superimposed on hypofluorescence lesions on ICGA $(M, N)$.

In the eye of patient 10 ('MFC with neovascularization'), OCT-A showed the CNV at the outer retina (I) as a hyper-reflective glomerule with a choriocapillary hyperreflective area (L). The superimposed ICGA showed an hypofluorescent lesion (O).

$(A, B, C, D, E, F)$ OCT-A showed normal superficial and deep capillary plexuses.

MFC: multifocal choroiditis

$68 \times 121 \mathrm{~mm}(150 \times 150 \mathrm{DPI})$ 


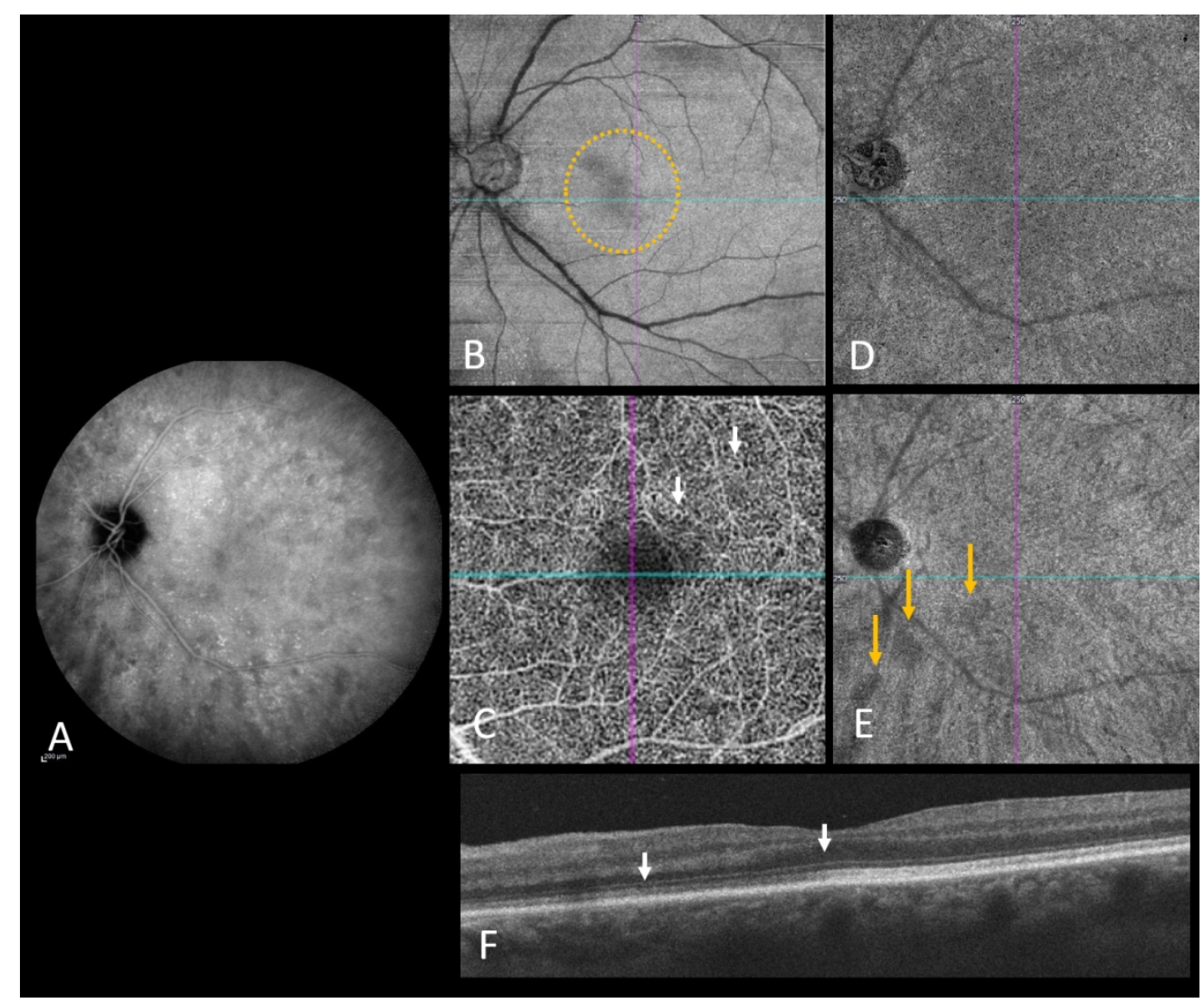

Figure 8: ICG showed widespread hypofluorescence dots in the left eye (A). En face OCT at the level of the IS/OS Ellipsoid showed decreased hyperreflectivity at the fovea (B). Foveal OCT-angiograms $6 \times 6 \mathrm{~mm}$ of retinal vasculature showed changes in deep capillary plexus (DCP) showing capillary loops (white arrows),

OCT-A does no showed choriocapillaris alteration in maculae (D) and choroid flow voids at the birdshot lesion inferior to the fovea and to the optic nerve (orange arrows (E). The SD-OCT scans shows diffuse slight reduction of the reflectivity of both the EZ and the IZ (area between arrows), although both are intact.IS/OS: inner segment/outer segment junction 


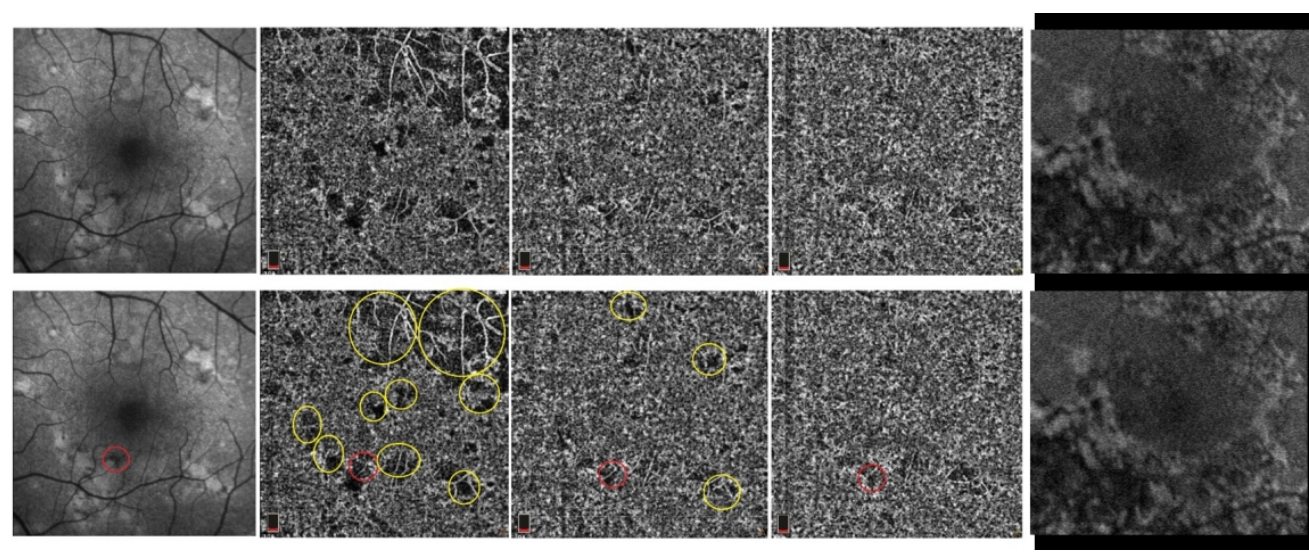




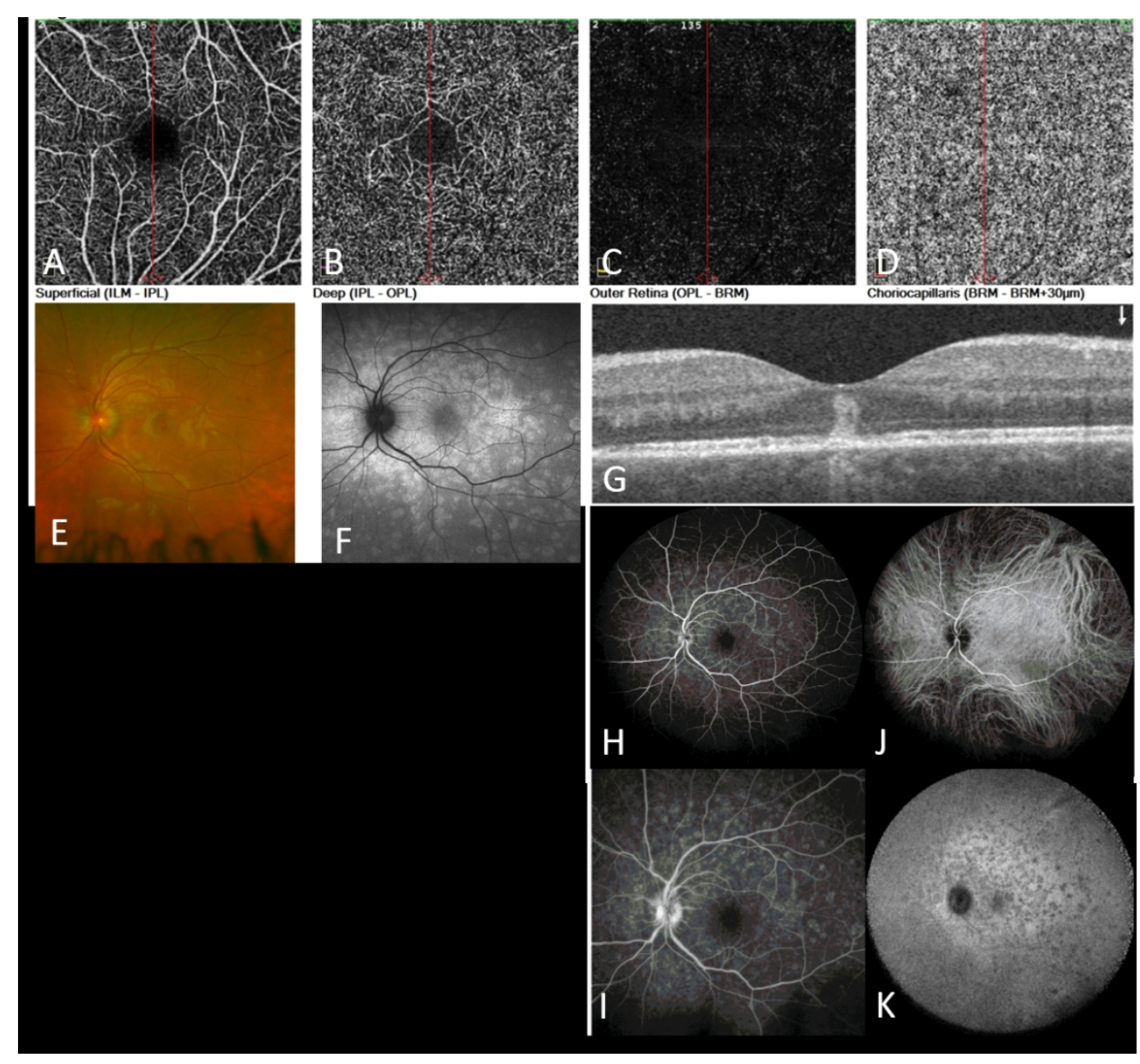

$199 \times 184 \mathrm{~mm}(150 \times 150 \mathrm{DPI})$ 




Figure 6'. SD-OCT and OCT Angiography of a patient with serpiginous choroiditis (Patient 7): $(A, B, C)$ OCT-A showed no flow abnormalities of the superficial $(A)$ and deep capillary

plexuses (B) and the outer retina (C). (D) There were flow impairment at the level of the choriocapillaris (yellow and red circles). Larger choroidal vessels have been pushed inward into the area of choriocapillaris alteration so are seen (red circles on OCT-A). (F)

Corresponding SD-OCT scan shows the loss of RPE causing increased intensity below Bruch's membrane which is characteristic RPE atrophy. (F) SD-OCT found areas with atrophy of the outer retina and ellipsoid disruption with retinal cysts regarding RPE atrophy (yellow arrow).

(E) These lesions associated with RPE atrophy appeared hypoautofluorescent on FAF (red circles) although other lesions were rather hyperautofluorescent (yellow circles). FAF: autofluorescence; RPE: Retinal Pigment Epithelium

$195 \times 185 \mathrm{~mm}(150 \times 150 \mathrm{DPI})$ 


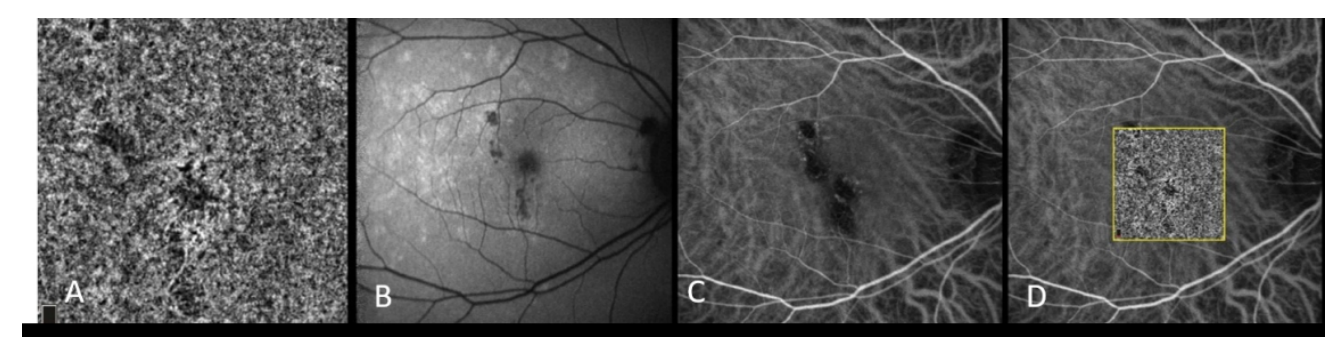

$240 \times 59 \mathrm{~mm}(150 \times 150 \mathrm{DPI})$ 


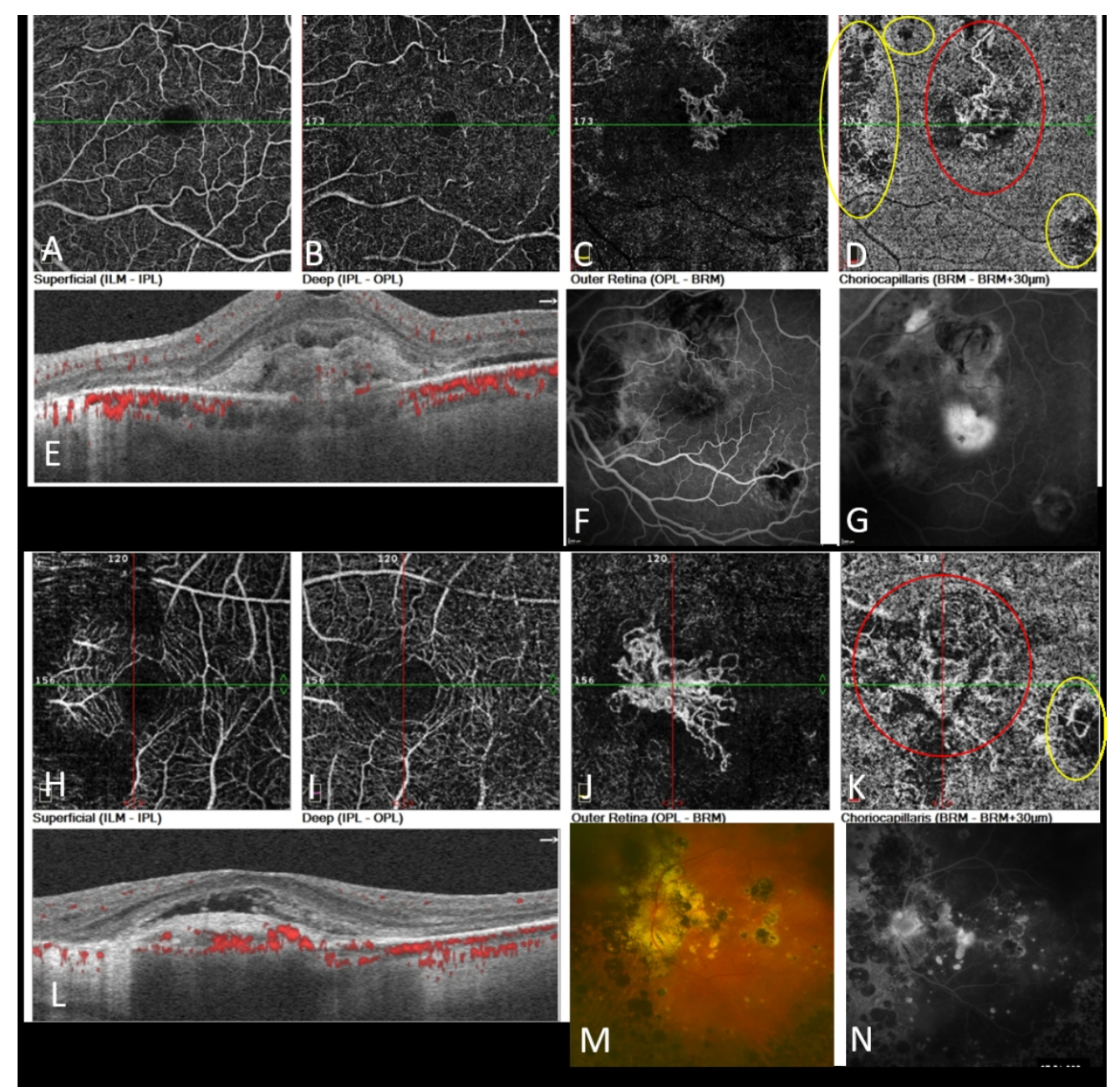

$201 \times 198 \mathrm{~mm}(150 \times 150 \mathrm{DPI})$ 

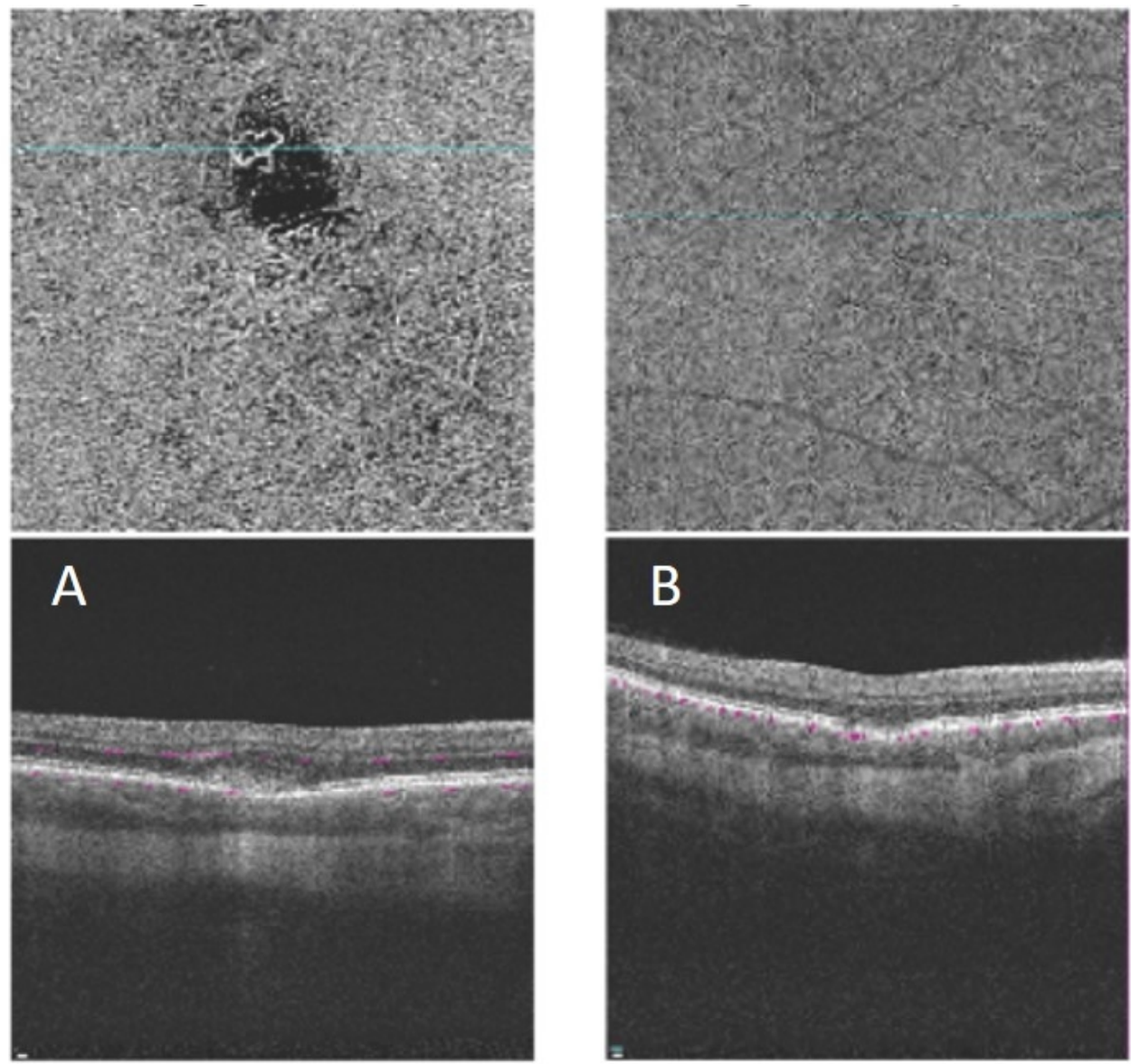

Figure 7"'. Patient 14 (A-B) with PIC and CNV: \%"OCT-A showed the CNV at the choriocapillaris (A, top) as a hyper-reflective glomerule area. On $B$, the CNV is no longer seen one month after anti-VEGF ocular injection. OCT (A) showed CNV without intraretinal or subretinal fluid.\%"MFC: punctate inner choroiditis; CNV: chorioretinal neovascularization\%"

$121 \times 109 \mathrm{~mm}(150 \times 150 \mathrm{DPI})$ 

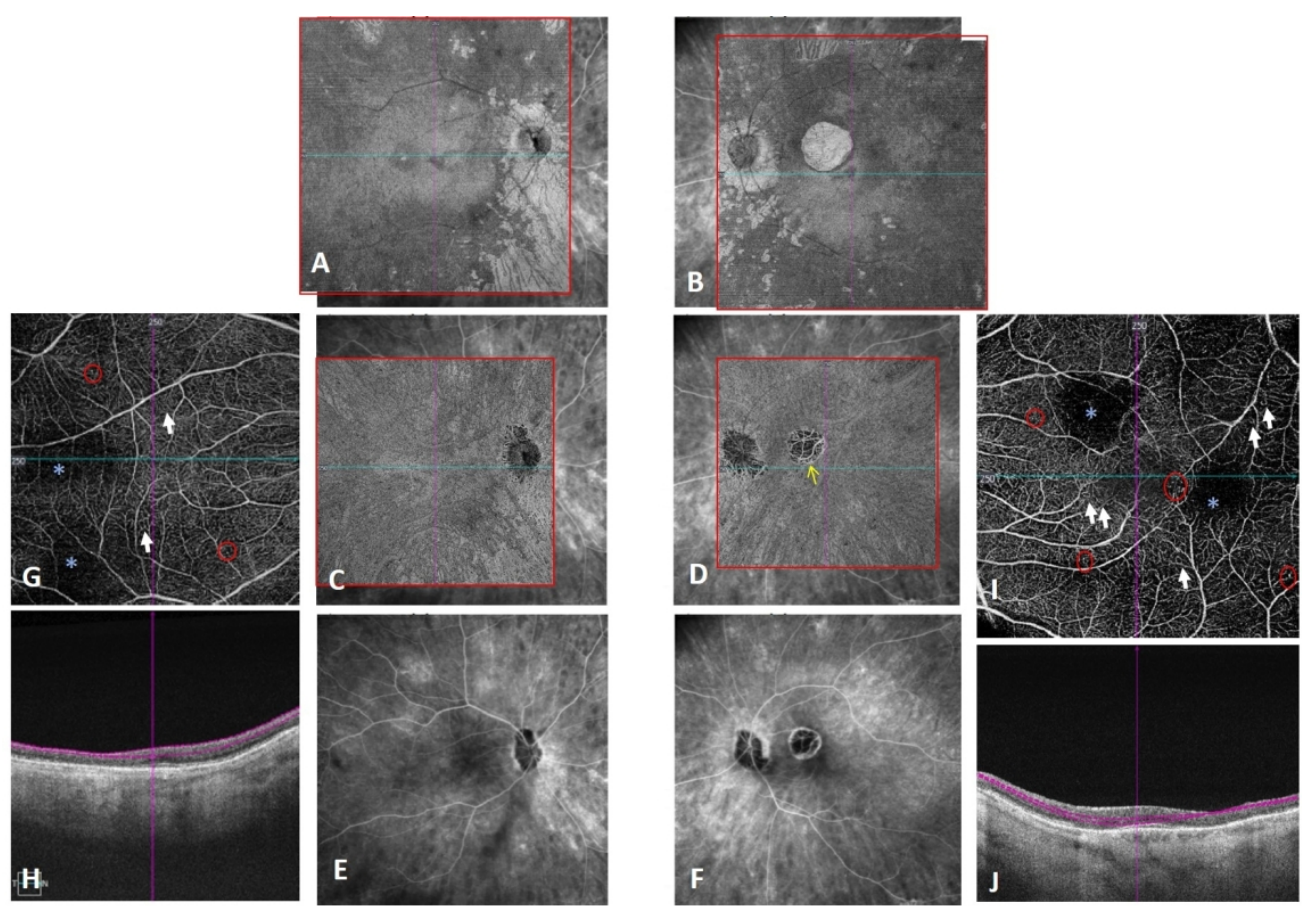

$297 \times 212 \mathrm{~mm}(150 \times 150 \mathrm{DPI})$

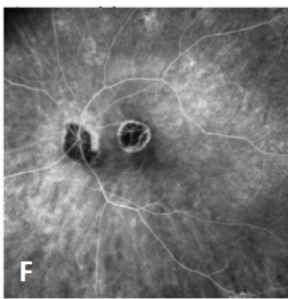




\begin{tabular}{|c|c|c|c|c|c|c|c|c|}
\hline patient & $\begin{array}{l}\text { Age, } \\
\text { gender }\end{array}$ & diagnosis & $\begin{array}{l}\text { Initial } \\
\text { BCVA }\end{array}$ & Imaging & $\begin{array}{l}\text { Follow-up } \\
\text { duration } \\
\text { at time of } \\
\text { 1st } \\
\text { imaging } \\
\text { (M) }\end{array}$ & $\begin{array}{l}\text { Final } \\
\text { BCVA }\end{array}$ & treatment & complication \\
\hline 1 & $M / 26$ & APMPPE & 20/50 LE & $\begin{array}{l}\text { FAF, } \\
\text { FA, ICG, } \\
\text { SD- } \\
\text { OCT, } \\
\text { OCT-A }\end{array}$ & 1 & $\begin{array}{l}20 / 20 \\
L E\end{array}$ & $\begin{array}{l}\text { PO steroids } 0.5 \\
\mathrm{mg} / \mathrm{kg} / \mathrm{d}\end{array}$ & none \\
\hline 2 & $M / 33$ & APMPPE & $20 / 100$ LE & $\begin{array}{l}\text { FA, ICG, } \\
\text { SD- } \\
\text { OCT, } \\
\text { OCT-A }\end{array}$ & 0.5 & $\begin{array}{l}20 / 40 \\
\mathrm{LE}\end{array}$ & $\begin{array}{l}\text { PO steroids } 0.5 \\
\mathrm{mg} / \mathrm{kg} / \mathrm{d}\end{array}$ & none \\
\hline 3 & $F / 21$ & MEWDS & $20 / 40 \mathrm{RE}$ & $\begin{array}{l}\text { FAF, } \\
\text { FA, ICG, } \\
\text { SD- } \\
\text { OCT, } \\
\text { OCT-A }\end{array}$ & $<0.5$ & $\begin{array}{l}20 / 25 \\
R E\end{array}$ & none & none \\
\hline 4 & $F / 15$ & MEWDS & $20 / 70$ LE & $\begin{array}{l}\text { Color } \\
\text { fundus, } \\
\text { FAF, } \\
\text { FA, ICG, } \\
\text { SD- } \\
\text { OCT, } \\
\text { OCT-A }\end{array}$ & $<0.5$ & $\begin{array}{l}20 / 20 \\
L E\end{array}$ & none & none \\
\hline 5 & $M / 25$ & MEWDS & 20/30 LE & $\begin{array}{l}\text { FAF, } \\
\text { FA, ICG, } \\
\text { SD- } \\
\text { OCT, } \\
\text { OCT-A }\end{array}$ & $<0.5$ & $\begin{array}{l}20 / 20 \\
L E\end{array}$ & none & none \\
\hline
\end{tabular}




\begin{tabular}{|c|c|c|c|c|c|c|c|c|}
\hline 6 & $M / 33$ & TB-SLC & $20 / 32$ & $\begin{array}{l}\text { FAF, } \\
\text { FA, ICG, } \\
\text { SD- } \\
\text { OCT, } \\
\text { OCT-A }\end{array}$ & 1 & $20 / 32$ & $\begin{array}{l}\text { PO steroids }+ \\
\text { quadritherapy } \\
\text { anti-TB }\end{array}$ & $\begin{array}{l}\text { Increase oral } \\
\text { steroids due } \\
\text { to } \\
\text { progression } \\
\text { of CR lesions }\end{array}$ \\
\hline 7 & $\mathrm{M} / 50$ & SC & $20 / 32$ LE & $\begin{array}{l}\text { FA, ICG, } \\
\text { SD- } \\
\text { OCT, } \\
\text { OCT-A }\end{array}$ & 1 & $\begin{array}{l}20 / 60 \\
\mathrm{LE}\end{array}$ & $\begin{array}{l}\text { PO steroids + } \\
\text { PO } \\
\text { azathioprine }\end{array}$ & none \\
\hline 8 & $F / 25$ & $\begin{array}{l}\text { MFC } \\
\text { without } \\
\text { CNV }\end{array}$ & $20 / 32 \mathrm{RE}$ & $\begin{array}{l}\text { FA, ICG, } \\
\text { SD- } \\
\text { OCT, } \\
\text { OCT-A }\end{array}$ & 1 & $\begin{array}{l}20 / 20 \\
\mathrm{RE}\end{array}$ & $\begin{array}{l}\text { IV solumedrol } \\
\text { and PO Pred. }\end{array}$ & none \\
\hline 9 & $F / 63$ & $\begin{array}{l}\text { MFC } \\
\text { with } \\
\text { CNV }\end{array}$ & $20 / 60$ & $\begin{array}{l}\text { FA, ICG, } \\
\text { SD- } \\
\text { OCT, } \\
\text { OCT-A }\end{array}$ & 2 & $\begin{array}{l}20 / 40 \\
\text { LE }\end{array}$ & $\begin{array}{l}\text { PSTK + anti- } \\
\text { VEGF }\end{array}$ & none \\
\hline 10 & $F / 26$ & $\begin{array}{l}\text { MFC } \\
\text { without } \\
\text { CNV }\end{array}$ & $20 / 40$ LE & $\begin{array}{l}\text { FA, ICG, } \\
\text { SD- } \\
\text { OCT, } \\
\text { OCT-A }\end{array}$ & 1.5 & $\begin{array}{l}20 / 30 \\
\mathrm{LE}\end{array}$ & $\begin{array}{l}\text { PSTK + } \\
\text { azathioprine }\end{array}$ & $\begin{array}{l}\text { azathioprine } \\
\text { intolerance }\end{array}$ \\
\hline 11 & $M / 23$ & $\begin{array}{l}\text { MFC } \\
\text { without } \\
\text { CNV }\end{array}$ & $20 / 32 \mathrm{RE}$ & $\begin{array}{l}\text { FA, ICG, } \\
\text { SD- } \\
\text { OCT, } \\
\text { OCT-A }\end{array}$ & $<0.5$ & $\begin{array}{l}20 / 32 \\
\mathrm{RE}\end{array}$ & Oral Pred. & none \\
\hline 12 & $\mathrm{~F} / 40$ & $\begin{array}{l}\text { MFC } \\
\text { with } \\
\text { CNV }\end{array}$ & $20 / 60 \mathrm{LE}$ & $\begin{array}{l}\text { FA, ICG, } \\
\text { SD- } \\
\text { OCT, } \\
\text { OCT-A }\end{array}$ & 6 & $\begin{array}{l}20 / 60 \\
L E\end{array}$ & $\begin{array}{l}\text { PO prednisone, } \\
\text { PO } \\
\text { methotrexate } \\
\text { 15mg weekly, } \\
\text { anti-VEGF }\end{array}$ & $\begin{array}{l}\text { Relapse of } \\
\text { CNV, switch } \\
\text { methotrexate } \\
\text { for } \\
\text { adalimumab. } \\
\text { Side effects } \\
\text { of } \\
\text { methotrexate }\end{array}$ \\
\hline
\end{tabular}




\begin{tabular}{|c|c|c|c|c|c|c|c|c|}
\hline 13 & $M / 63$ & $\begin{array}{l}\text { MFC } \\
\text { with } \\
\text { CNV }\end{array}$ & $20 / 60$ OS & $\begin{array}{l}\text { FAF, } \\
\text { FA, SD- } \\
\text { OCT, } \\
\text { OCT-A }\end{array}$ & 5 & $\begin{array}{l}20 / 100 \\
\text { OS }\end{array}$ & $\begin{array}{l}\text { PSTK } 1 \text { month } \\
\text { ago, repeated } \\
\text { anti-VEGF }\end{array}$ & $\begin{array}{l}\text { Need for } \times 6 \\
\text { anti-VEGF, } \\
\text { then } \\
\text { adalimumab }\end{array}$ \\
\hline 14 & $M / 30$ & $\begin{array}{l}\text { PIC with } \\
\text { CNV }\end{array}$ & $20 / 20 \mathrm{RE}$ & $\begin{array}{l}\text { Color } \\
\text { fundus, } \\
\text { FAF, } \\
\text { SD- } \\
\text { OCT, } \\
\text { OCT-A }\end{array}$ & $\begin{array}{l}<0.5 . \\
\text { Active } \\
\text { choroiditis } \\
\text { diagnosed } \\
3 \text { months } \\
\text { ago }\end{array}$ & $\begin{array}{l}20 / 20 \\
\mathrm{RE}\end{array}$ & $\begin{array}{l}\text { anti-VEGF, } \\
\text { increase PO } \\
\text { Pred. to } \\
0.5 \mathrm{mg} / \mathrm{Kg} / \text { daily. } \\
\text { Pt on PO Pred. } \\
10 \mathrm{mg} \text { and PO } \\
\text { methotrexate } \\
20 \mathrm{mg} \text { weekly } \\
\text { for recent } \\
\text { choroiditis }\end{array}$ & none \\
\hline 15 & $F / 66$ & BSCR & $\begin{array}{l}\text { 20/50 BE } \\
\text { (LE } \\
\text { amblyopia) }\end{array}$ & $\begin{array}{l}\text { Color } \\
\text { fundus, } \\
\text { FAF, } \\
\text { FA, ICG, } \\
\text { SD- } \\
\text { OCT, } \\
\text { OCT-A }\end{array}$ & 6 & $\begin{array}{l}20 / 30 \\
R E \\
20 / 40 \\
L E\end{array}$ & $\begin{array}{l}\text { Repeated } \\
\text { intravitreal } \\
\text { triamcinolone } \\
\text { injections } \mathrm{BE} \text {, } \\
\text { plan: for } \\
\text { fluocinolone } \\
\text { acetonide } \\
\text { intravitreal } \\
\text { implant } \mathrm{BE}\end{array}$ & $\begin{array}{l}\text { Lenses } \\
\text { opacifications }\end{array}$ \\
\hline 16 & $F / 72$ & BSCR & unknown & $\begin{array}{l}\text { Color } \\
\text { fundus, } \\
\text { FAF, } \\
\text { FA, ICG, } \\
\text { SD- } \\
\text { OCT, } \\
\text { OCT-A, } \\
\text { ERG }\end{array}$ & 144 & $\begin{array}{l}20 / 40 \\
R E \\
20 / 30 \\
L E\end{array}$ & $\begin{array}{l}\text { Mycophenolate } \\
\text { mofetil PO(1.5 } \\
\text { gram daily), PO } \\
\text { Pred. } 2.5 \mathrm{mg} \\
\text { daily }\end{array}$ & $\begin{array}{l}\text { Steroids } \\
\text { responder, } \\
\text { antiglaucoma } \\
\text { eye drops BE }\end{array}$ \\
\hline
\end{tabular}


BE: both eyes, birdshot chorioretinopathy (BSCR), CR: chorioretinal, CNV: chorioretinal neovascularization, ERG: electrophysiology, FAF: fundus autofluorescence FAF, LE: left eye, M: Months, multifocal choroiditis (MFC), multiple evanescent white dot syndrome (MEWDS), Pred. : prednisolone, PO: per os, PSTK: subtenon triamcinolone injection, Pt: patient, punctate inner choroiditis (PIC), RE: right eye,

serpiginous choroiditis (SC), tuberculosis (TB) -related serpiginous-like choroiditis (SLC) 


\begin{tabular}{|c|c|c|c|c|}
\hline patient & diagnosis & Imaging & Initial OCT-A findings & Final OCT-A findings \\
\hline 1 & APMPPE & $\begin{array}{l}\text { FAF, } \\
\text { FA, ICG, } \\
\text { SD- } \\
\text { OCT, } \\
\text { OCT-A }\end{array}$ & $\begin{array}{l}\text { areas of hypoperfusion of } \\
\text { the choriocapillaris }\end{array}$ & $\begin{array}{l}\text { one lesion fails to recover located at the level of RPE and } \\
\text { choriocapillaris. The persistent lesion on OCT-A does not } \\
\text { show up on final FAF at } 2 \text { months }\end{array}$ \\
\hline 2 & APMPPE & $\begin{array}{l}\text { FA, ICG, } \\
\text { SD- } \\
\text { OCT, } \\
\text { OCT-A }\end{array}$ & $\begin{array}{l}\text { OCT-A revealed areas of hypoperfusion of } \\
\text { the choriocapillaris }\end{array}$ & NP \\
\hline 3 & MEWDS & $\begin{array}{l}\text { FAF, } \\
\text { FA, ICG, } \\
\text { SD- } \\
\text { OCT, } \\
\text { OCT-A }\end{array}$ & $\begin{array}{l}\text { OCT-A showed no flow abnormalities at superficial, } \\
\text { deep capillary plexus, outer retina and at } \\
\text { choriocapillaris levels }\end{array}$ & NP \\
\hline 4 & MEWDS & $\begin{array}{l}\text { Color } \\
\text { fundus, } \\
\text { FAF, } \\
\text { FA, ICG, } \\
\text { SD- } \\
\text { OCT, } \\
\text { OCT-A }\end{array}$ & $\begin{array}{l}\text { no flow abnormalities at superficial, deep capillary } \\
\text { plexus, outer retina and at choriocapillaris levels. }\end{array}$ & NP \\
\hline 5 & MEWDS & $\begin{array}{l}\text { FAF, } \\
\text { FA, ICG, } \\
\text { SD- } \\
\text { OCT, } \\
\text { OCT-A }\end{array}$ & $\begin{array}{l}\text { no flow abnormalities at superficial, deep capillary } \\
\text { plexus, outer retina and at choriocapillaris levels }\end{array}$ & NP \\
\hline 6 & TB-SLC & $\begin{array}{l}\text { FAF, } \\
\text { FA, ICG, } \\
\text { SD- } \\
\text { OCT, } \\
\text { OCT-A }\end{array}$ & $\begin{array}{l}\text { hypoperfusion areas of the choriocapillaris larger } \\
\text { than the lesions at the level of the RPE on FA }\end{array}$ & NP \\
\hline
\end{tabular}




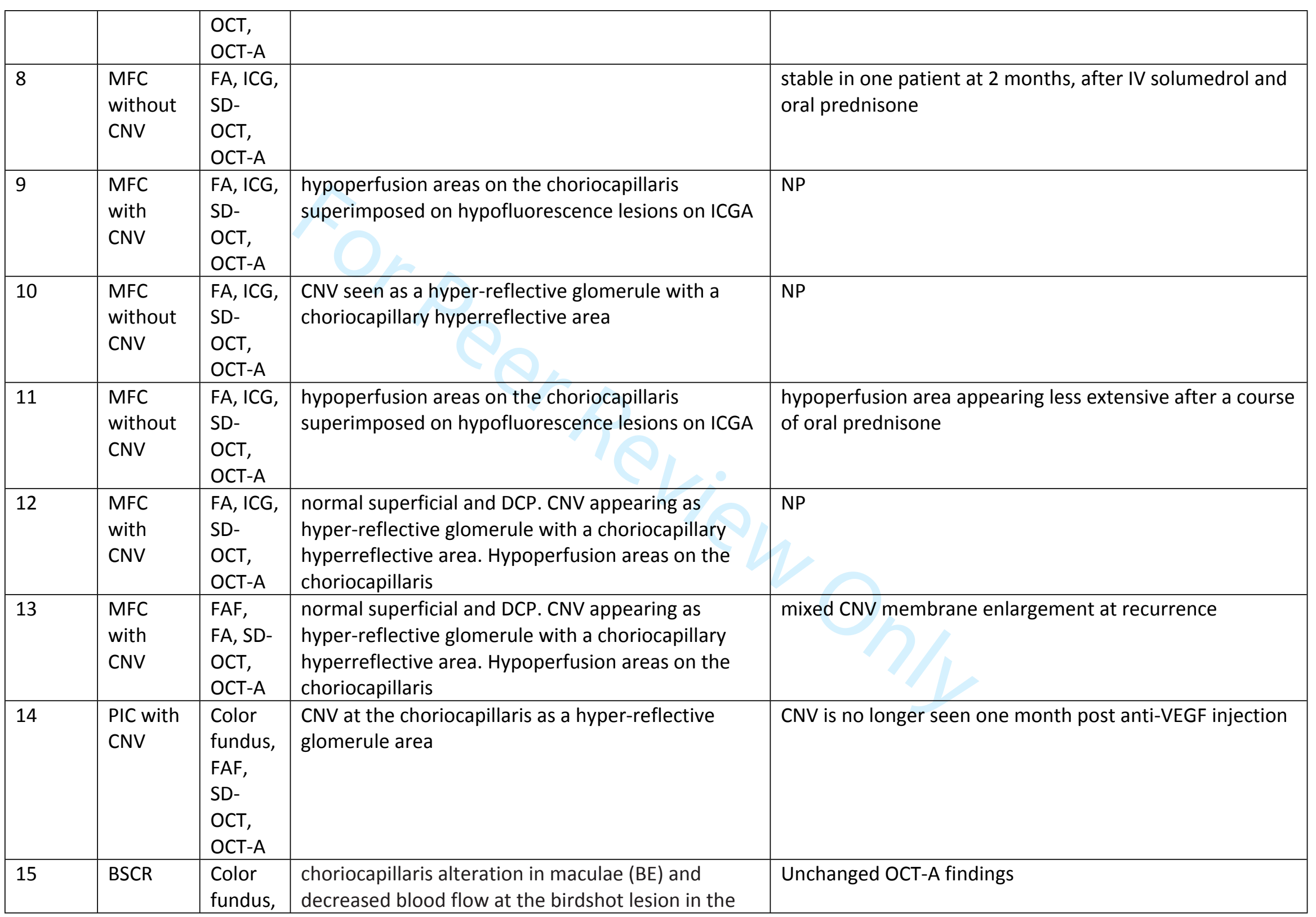




\begin{tabular}{|c|c|c|c|c|}
\hline & & $\begin{array}{l}\text { FAF, } \\
\text { FA, ICG, } \\
\text { SD- } \\
\text { OCT, } \\
\text { OCT-A }\end{array}$ & $\begin{array}{l}\text { fovea (LE). Larger choroidal vessels bordering the } \\
\text { birdshot lesion. } \\
\text { Retinal vasculature changes in SCP with } \\
\text { telangiectatic vessels, capillary loops, abnormally } \\
\text { increased intercapillary spaces or rather non- } \\
\text { perfused areas }\end{array}$ & \\
\hline 16 & BSCR & $\begin{array}{l}\text { Color } \\
\text { fundus, } \\
\text { FAF, } \\
\text { FA, ICG, } \\
\text { SD- } \\
\text { OCT, } \\
\text { OCT-A, } \\
\text { ERG }\end{array}$ & $\begin{array}{l}\text { changes in DCP: showing capillary loops, } \\
\text { no choriocapillaris alteration in maculae (BE) and } \\
\text { choroid flow voids at the birdshot lesion inferior to } \\
\text { the fovea and to the optic nerve }\end{array}$ & Unchanged OCT-A findings \\
\hline
\end{tabular}

BE: both eyes, birdshot chorioretinopathy (BSCR), CNV: chorioretinal neovascularization deep capillary plexus (DCP), ERG: electrophysiology, FAF: fundus autofluorescence FAF, LE: left eye, M: Months, multifocal choroiditis (MFC), multiple evanescent white dot syndrome (MEWDS), punctate inner choroiditis (PIC), RE: right eye, SCP: superficial capillary plexus, serpiginous choroiditis (SC), tuberculosis (TB) -related serpiginouslike choroiditis (SLC) 